\title{
THE STRUCTURE OF SALLY MODULES AND BUCHSBAUMNESS OF ASSOCIATED GRADED RINGS
}

\author{
KAZUHO OZEKI
}

\section{To Shiro Goto on the occasion of his sixty-fifth birthday}

\begin{abstract}
Let $A$ be a Noetherian local ring with the maximal ideal $\mathfrak{m}$, and let $I$ be an $\mathfrak{m}$-primary ideal in $A$. This paper examines the equality on Hilbert coefficients of $I$ first presented by Elias and Valla, but without assuming that $A$ is a Cohen-Macaulay local ring. That equality is related to the Buchsbaumness of the associated graded ring of $I$.
\end{abstract}

\section{$\S 1$. Introduction and the statement of main results}

Throughout this paper, let $A$ denote a Noetherian local ring with the maximal ideal $\mathfrak{m}$, and let $d=\operatorname{dim} A>0$. For simplicity, we assume that the residue class field $A / \mathfrak{m}$ is infinite. Let $I$ be an $\mathfrak{m}$-primary ideal in $A$, and suppose that $I$ contains a parameter ideal $Q=\left(a_{1}, a_{2}, \ldots, a_{d}\right)$ of $A$ as a reduction - that is, $Q \subseteq I$ - and that the equality $I^{n+1}=Q I^{n}$ holds true for some (and hence any) integer $n \gg 0$. Let $\ell_{A}(M)$ denote, for an $A$-module $M$, the length of $M$. Then we have integers $\left\{\mathrm{e}_{i}(I)\right\}_{0 \leq i \leq d}$ such that the equality

$$
\ell_{A}\left(A / I^{n+1}\right)=\mathrm{e}_{0}(I)\left(\begin{array}{c}
n+d \\
d
\end{array}\right)-\mathrm{e}_{1}(I)\left(\begin{array}{c}
n+d-1 \\
d-1
\end{array}\right)+\cdots+(-1)^{d} \mathrm{e}_{d}(I)
$$

holds true for all integers $n \gg 0$, which we call the Hilbert coefficients of $A$ with respect to $I$.

Let

$$
R=\mathrm{R}(I):=A[I t] \quad \text { and } \quad T=\mathrm{R}(Q):=A[Q t] \subseteq A[t]
$$

denote, respectively, the Rees algebras of $I$ and $Q$. Put $F=T / I T$, and let

$$
R^{\prime}=\mathrm{R}^{\prime}(I):=A\left[I t, t^{-1}\right] \subseteq A\left[t, t^{-1}\right]
$$

Received July 12, 2011. Revised June 14, 2012. Accepted August 30, 2012.

First published online September 3, 2013.

2010 Mathematics Subject Classification. Primary 13D40; Secondary 13A30, 13 H10. 
and

$$
G=\mathrm{G}(I):=R^{\prime} / t^{-1} R^{\prime} \cong \bigoplus_{n \geq 0} I^{n} / I^{n+1} .
$$

Following Vasconcelos [23], we then define

$$
S=\mathrm{S}_{Q}(I):=I R / I T \cong \bigoplus_{n \geq 1} I^{n+1} / Q^{n} I
$$

and call it the Sally module of $I$ with respect to $Q$.

In the case where $A$ is a Cohen-Macaulay local ring, we have the inequality

$$
2 \mathrm{e}_{0}(I)-\mathrm{e}_{1}(I) \leq 2 \ell_{A}(A / I)+\ell_{A}\left(I / I^{2}+Q\right)
$$

which is given in [3, Theorem 2.1] and [11, Proposition 2.1], and they showed that the equality $2 \mathrm{e}_{0}(I)-\mathrm{e}_{1}(I)=2 \ell_{A}(A / I)+\ell_{A}\left(I / I^{2}+Q\right)$ holds true if and only if $I^{3}=Q I^{2}$ and $Q \cap I^{2}=Q I$. When this is the case, the associated graded ring $G$ of $I$ is Cohen-Macaulay. Thus, the ideal $I$ with $2 \mathrm{e}_{0}(I)-\mathrm{e}_{1}(I)=2 \ell_{A}(A / I)+\ell_{A}\left(I / I^{2}+Q\right)$ has nice properties. The purpose of this paper is to extend their results without assuming that $A$ is a CohenMacaulay ring.

In an arbitrary Noetherian local ring $A$, the inequality

$$
2 \mathrm{e}_{0}(I)-\mathrm{e}_{1}(I)+\mathrm{e}_{1}(Q) \leq 2 \ell_{A}(A / I)+\ell_{A}\left(I / I^{2}+Q\right)
$$

holds true (see [18, Theorem 2.4], [1, Theorem 3.1]). It seems natural to ask what happens on the ideals $I$ satisfying the equality $2 \mathrm{e}_{0}(I)-\mathrm{e}_{1}(I)+\mathrm{e}_{1}(Q)=$ $2 \ell_{A}(A / I)+\ell_{A}\left(I / I^{2}+Q\right)$.

Corso in [1] conjectured that the associated graded ring $G(\mathfrak{m})$ of the maximal ideal $\mathfrak{m}$ is a Buchsbaum ring if $A$ is a Buchsbaum local ring and if the equality $2 \mathrm{e}_{0}(\mathfrak{m})-\mathrm{e}_{1}(\mathfrak{m})+\mathrm{e}_{1}(Q)=v(A)-d+2$ holds true for $\mathfrak{m}$ and a reduction $Q$ of $\mathfrak{m}$, where $v(A)$ denotes the embedding dimension of $A$, and he gave a positive answer to this conjecture in 1-dimensional cases. We should note that Rossi and Valla [18, Theorem 2.1] generalized this result in 1-dimensional Buchsbaum module cases. The current author, in [16, Theorem 1.2], gave an affirmative answer to Corso's conjecture for the case where $A$ is a Buchsbaum local ring with an arbitrary dimension.

To state the results of this article, let us consider the following four conditions.

$\left(\mathrm{C}_{0}\right)$ The sequence $a_{1}, a_{2}, \ldots, a_{d}$ is a $d$-sequence in $A$ in the sense of Huneke [13]. 
$\left(\mathrm{C}_{1}\right)$ The sequence $a_{1}, a_{2}, \ldots, a_{d}$ is a $d^{+}$-sequence in $A$; that is, for all integers $n_{1}, n_{2}, \ldots, n_{d} \geq 1$, the sequence $a_{1}^{n_{1}}, a_{2}^{n_{2}}, \ldots, a_{d}^{n_{d}}$ forms a $d$ sequence in any order.

$\left(\mathrm{C}_{2}\right)$ We have $\left(a_{1}, a_{2}, \ldots, \check{a}_{i}, \ldots, a_{d}\right):_{A} a_{i} \subseteq I$ for all $1 \leq i \leq d$.

$\left(\mathrm{C}_{3}\right)$ We have $\operatorname{depth} A>0$.

These conditions $\left(\mathrm{C}_{0}\right),\left(\mathrm{C}_{1}\right),\left(\mathrm{C}_{2}\right)$, and $\left(\mathrm{C}_{3}\right)$ are naturally satisfied when $A$ is a Cohen-Macaulay local ring. Condition $\left(\mathrm{C}_{1}\right)$ (resp., condition $\left(\mathrm{C}_{2}\right)$ ) is always satisfied if $A$ is a Buchsbaum local ring (resp., $I=\mathfrak{m}$ ). Here we notice that condition $\left(\mathrm{C}_{1}\right)$ is equivalent to saying that our local ring $A$ is a generalized Cohen-Macaulay ring; that is, all the local cohomology modules $\mathrm{H}_{\mathfrak{m}}^{i}(A)$ $(i \neq d)$ of $A$ with respect to the maximal ideal $\mathfrak{m}$ are finitely generated, and the parameter ideal $Q$ is standard. In other words, the equality

$$
\ell_{A}(A / Q)-\mathrm{e}_{0}(Q)=\sum_{i=0}^{d-1}\left(\begin{array}{c}
d-1 \\
i
\end{array}\right) \cdot \ell_{A}\left(\mathrm{H}_{\mathfrak{m}}^{i}(A)\right)
$$

holds true. Hence, condition $\left(\mathrm{C}_{1}\right)$ is independent of the choice of a minimal system $\left\{a_{i}\right\}_{1 \leq i \leq d}$ of generators of the parameter ideal $Q$. We note here that condition $\left(\mathrm{C}_{2}\right)$ is also independent of the choice of a minimal system $\left\{a_{i}\right\}_{1 \leq i \leq d}$ of generators of $Q$.

Let us now state our own result. The main result of this article is the following Theorem 1.1, which generalizes the results of [3, Theorem 2.1] and [11, Theorem 2.2] given in the case where $A$ is a Cohen-Macaulay local ring, because $\mathrm{e}_{i}(Q)=0$ for all $1 \leq i \leq d$. We notice that, thanks to condition $\left(\mathrm{C}_{1}\right)$, the Hilbert coefficients $\mathrm{e}_{i}(Q)$ of $Q$ are given by the formula

$$
(-1)^{i} \mathrm{e}_{i}(Q)= \begin{cases}\mathrm{e}_{0}(Q) & \text { if } i=0, \\
\ell_{A}\left(\mathrm{H}_{\mathfrak{m}}^{0}(A)\right) & \text { if } i=d, \\
\sum_{j=1}^{d-i}\left(\begin{array}{c}
d-i-1 \\
j-1
\end{array}\right) \ell_{A}\left(\mathrm{H}_{\mathfrak{m}}^{j}(A)\right) & \text { if } 1 \leq i \leq d-1,\end{cases}
$$

and one has the equality $\ell_{A}\left(A / Q^{n+1}\right)=\sum_{i=0}^{d}(-1)^{i} \mathrm{e}_{i}(Q)\left(\begin{array}{c}n+d-i \\ d-i\end{array}\right)$ for all $n \geq 0$ (see [19, Korollar 3.2]), so that the $\left\{\mathrm{e}_{i}(Q)\right\}_{1 \leq i \leq d}$ are independent of the choice of the reduction $Q$ of $I$ and so are invariants of $A$. Here, $W=\mathrm{H}_{\mathfrak{m}}^{0}(A)$ denotes the 0 th local cohomology modules of $A$ with respect to $\mathfrak{m}$, and $\mathrm{H}_{M}^{i}(G)$ denotes the $i$ th local cohomology modules of $G$ with respect to the graded maximal ideal $M=\mathfrak{m} T+T_{+}$of $T$.

TheOREM 1.1. Suppose that conditions $\left(\mathrm{C}_{1}\right)$ and $\left(\mathrm{C}_{2}\right)$ are satisfied. Then the following two conditions are equivalent: 
(1) $2 \mathrm{e}_{0}(I)-\mathrm{e}_{1}(I)+\mathrm{e}_{1}(Q)=2 \ell_{A}(A / I)+\ell_{A}\left(I / I^{2}+Q\right)$;

(2) $I^{3} \subseteq Q I^{2}+W,\left(Q^{n}+W\right) \cap\left(I^{n+1}+W\right)=Q^{n} I+W$ for all $n \geq 1$, and $\left(a_{1}, a_{2}, \ldots, \check{a_{i}}, \ldots, a_{d}\right):_{A} a_{i} \subseteq I^{2}+Q$ for all $1 \leq i \leq d$.

When this is the case, we have $I^{2} \supseteq W$, and the following assertions also hold true.

(i) For all $n \in \mathbb{Z}$,

$$
\left[\mathrm{H}_{M}^{0}(G)\right]_{n} \cong \begin{cases}W / I^{3} \cap W & \text { if } n=2, \\ I^{n} \cap W / I^{n+1} \cap W & \text { if } n \geq 3 \\ (0) & \text { otherwise. }\end{cases}
$$

Hence, $\left[\mathrm{H}_{M}^{0}(G)\right]_{2} \cong W / I^{3} \cap W,\left[\mathrm{H}_{M}^{0}(G)\right]_{3} \cong I^{3} \cap W$, and $\left[\mathrm{H}_{M}^{0}(G)\right]_{n}=$ (0) for all $n \neq 2,3$ if $A$ is a Buchsbaum local ring.

(ii) We have

$$
\mathrm{H}_{M}^{i}(G)=\left[\mathrm{H}_{M}^{i}(G)\right]_{2-i} \cong \mathrm{H}_{\mathfrak{m}}^{i}(A)
$$

for all $1 \leq i \leq d-1$.

(iii) The a-invariant

$$
\mathrm{a}(G):=\max \left\{n \in \mathbb{Z} \mid\left[\mathrm{H}_{M}^{d}(G)\right]_{n} \neq(0)\right\}
$$

of $G$ is at most $2-d$.

(iv) We have $\mathrm{e}_{2}(I)=\mathrm{e}_{1}(Q)+\mathrm{e}_{2}(Q)-\mathrm{e}_{0}(I)+\mathrm{e}_{1}(I)+\ell_{A}(A / I)$ if $d \geq 2$.

(v) We have $\mathrm{e}_{i}(I)=\mathrm{e}_{i-2}(Q)+2 \mathrm{e}_{i-1}(Q)+\mathrm{e}_{i}(Q)$ for all $3 \leq i \leq d$.

(vi) The associated graded ring $G$ is a Buchsbaum ring with the Buchsbaum invariant $\mathbb{I}(G)=\mathbb{I}(A)$ if $A$ is a Buchsbaum local ring.

The key of the proof of Theorem 1.1 is the use of the Sally module $S$ of $I$ with respect to $Q$. In [16], the author used some techniques of [1] and [2] concerning the Sally module $\mathrm{S}_{Q}(\mathfrak{m})$ of the maximal ideal $\mathfrak{m}$ with respect to a reduction $Q$ of $\mathfrak{m}$ and gave the Buchsbaumness of the associated graded ring $\mathrm{G}(\mathfrak{m})$ of $\mathfrak{m}$ satisfying the equality $2 \mathrm{e}_{0}(\mathfrak{m})-\mathrm{e}_{1}(\mathfrak{m})+\mathrm{e}_{1}(Q)=v(A)-d+2$ in a Buchsbaum local ring $(A, \mathfrak{m})$. In this paper, to prove Theorem 1.1 , we will make some improvements to the techniques of [1] and [2] for the case where $I$ is an $\mathfrak{m}$-primary ideal (not necessary maximal ideal).

We are now in a position to briefly explain how we organized this paper. We prove Theorem 1.1 in Sections 4 and 5. In Section 2, we summarize some auxiliary results on Sally modules for later use in this article. In Section 3, we introduce some techniques of Sally modules which are key for the proof of Theorem 1.1. In Section 4 we give a proof of the equivalence of conditions 
(1) and (2) in Theorem 1.1, and in Section 5 we give the last assertions in Theorem 1.1. In Section 6 , we give one example of an $\mathfrak{m}$-primary ideal $I$ with $2 \mathrm{e}_{0}(I)-\mathrm{e}_{1}(I)+\mathrm{e}_{1}(Q)=2 \ell_{A}(A / I)+\ell_{A}\left(I / I^{2}+Q\right)$ in a Buchsbaum local ring $(A, \mathfrak{m})$.

In what follows, unless otherwise specified, let $A$ be a Noetherian local ring with maximal ideal $\mathfrak{m}$, and let $d=\operatorname{dim} A>0$. Assume that the residue class field $A / \mathfrak{m}$ of $A$ is infinite. Let $I$ be an $\mathfrak{m}$-primary ideal in $A$, and put $Q=\left(a_{1}, a_{2}, \ldots, a_{d}\right)$ a parameter ideal of $A$ which forms a reduction of $I$. We put

$$
\begin{aligned}
& R=A[I t], \quad T=A[Q t], \quad R^{\prime}=A\left[I t, t^{-1}\right], \\
& G=R^{\prime} / t^{-1} R^{\prime}, \quad \text { and } \quad F=T / I T .
\end{aligned}
$$

We denote by $\mathrm{H}_{\mathfrak{m}}^{i}(*) \quad(i \in \mathbb{Z})$ the $i$ th local cohomology functor of $A$ with respect to $\mathfrak{m}$. Let $\mathbb{I}(A)=\sum_{i=0}^{d-1}\left(\begin{array}{c}d-1 \\ i\end{array}\right) \ell_{A}\left(\mathrm{H}_{\mathfrak{m}}^{i}(A)\right)$ denote the Buchsbaum invariant of $A$. Let $M=\mathfrak{m} T+T_{+}$be the unique graded maximal ideal in $T$. We denote by $\mathrm{H}_{M}^{i}(*)(i \in \mathbb{Z})$ the $i$ th local cohomology functor of $T$ with respect to $M$, and we let $\mathbb{I}(G)=\sum_{i=0}^{d-1}\left(\begin{array}{c}d-1 \\ i\end{array}\right) \ell_{G}\left(\mathrm{H}_{M}^{i}(G)\right)$ be the Buchsbaum invariant of $G$. Let $L$ be a graded $T$-module. We denote by $L(\alpha)$, for each $\alpha \in \mathbb{Z}$, the graded $T$-module whose grading is given by $[L(\alpha)]_{n}=[L]_{\alpha+n}$ for all $n \in \mathbb{Z}$. Let $\mu_{A}(I)$ denote the number of a minimal system of generators of $I$.

\section{$\S 2$. The structure of Sally modules}

In our proof of Theorem 1.1, we need some structure theorems of Sally modules.

The purpose of this section is to summarize some auxiliary results on Sally modules, which we need throughout this paper (see [7], [8], and [23] for the detailed proofs).

REMARK 2.1 (see [7], [8], [23]). We notice that $S$ is a finitely generated graded $T$-module and that $\mathfrak{m}^{\ell} \cdot S=(0)$ for some integer $\ell \gg 0$. Since $R$ is a module finite extension of the graded $\operatorname{ring} T$ and since $\mathfrak{m}=\sqrt{Q}, \operatorname{dim}_{T} S \leq d$. The following assertions also follow:

(1) $S=(0)$ if and only if $I^{2}=Q I$,

(2) $I S=(0)$ if and only if $I^{n+2} \subseteq Q^{n} I$ for all $n \geq 0$.

Lemma 2.2. Suppose that conditions $\left(\mathrm{C}_{0}\right)$ and $\left(\mathrm{C}_{2}\right)$ are satisfied. Then

$$
F=T / I T \cong(A / I)\left[X_{1}, X_{2}, \ldots, X_{d}\right]
$$


as graded $A$-algebras, where $(A / I)\left[X_{1}, X_{2}, \ldots, X_{d}\right]$ denotes the polynomial ring with $d$ indeterminates over the Artinian local ring $A / I$.

Proof. See [8, Proposition 2.2].

Let us note the following lemma. We notice that Serre's condition $\left(\mathrm{S}_{2}\right)$ on $T$ is automatically satisfied, once both conditions $\left(\mathrm{C}_{1}\right)$ and $\left(\mathrm{C}_{3}\right)$ are satisfied (see [21, Theorem 6.2]).

Lemma 2.3. Suppose that conditions $\left(\mathrm{C}_{0}\right),\left(\mathrm{C}_{2}\right)$, and $\left(\mathrm{C}_{3}\right)$ are satisfied. Assume that the ring $T$ satisfies Serre's condition $\left(\mathrm{S}_{2}\right)$. Then $\operatorname{Ass}_{T} S \subseteq$ $\{\mathfrak{m} T\}$, whence $\operatorname{dim}_{T} S=d$ if $S \neq(0)$.

Proof. See [8, Lemma 2.3].

Proposition 2.4. Suppose that conditions $\left(\mathrm{C}_{0}\right)$ and $\left(\mathrm{C}_{2}\right)$ are satisfied. Then

$$
\begin{aligned}
\ell_{A}\left(A / I^{n+1}\right)= & \mathrm{e}_{0}(I)\left(\begin{array}{c}
n+d \\
d
\end{array}\right)-\left\{\mathrm{e}_{0}(I)+\mathrm{e}_{1}(Q)-\ell_{A}(A / I)\right\}\left(\begin{array}{c}
n+d-1 \\
d-1
\end{array}\right) \\
& +\sum_{i=2}^{d}(-1)^{i}\left\{\mathrm{e}_{i-1}(Q)+\mathrm{e}_{i}(Q)\right\}\left(\begin{array}{c}
n+d-i \\
d-i
\end{array}\right)-\ell_{A}\left(S_{n}\right)
\end{aligned}
$$

for all $n \geq 0$.

Proof. Our condition $\left(\mathrm{C}_{0}\right)$ implies that $\ell_{A}\left(A / Q^{n+1}\right)=\sum_{i=0}^{d} \mathrm{e}_{i}(Q)\left(\begin{array}{c}n+d-i \\ d-i\end{array}\right)$ for all $n \geq 0$ (see [9, Proposition 3.4]). Hence, by [8, Proposition 2.4], we get a required condition.

Put $s=\operatorname{dim}_{T} S \leq d$. Then we write

$$
\ell_{A}\left(S_{n}\right)=\mathrm{e}_{0}(S)\left(\begin{array}{c}
n+s-1 \\
s-1
\end{array}\right)-\mathrm{e}_{1}(S)\left(\begin{array}{c}
n+s-2 \\
s-2
\end{array}\right)+\cdots+(-1)^{s-1} \mathrm{e}_{s-1}(S)
$$

for all $n \gg 0$ with integers $\left\{\mathrm{e}_{i}(S)\right\}_{0 \leq i \leq s-1}$. Then by Proposition 2.4 we get the following result, which is also given in [18, Proposition 6.2].

Corollary 2.5. Suppose that conditions $\left(\mathrm{C}_{0}\right)$ and $\left(\mathrm{C}_{2}\right)$ are satisfied, and put $s=\operatorname{dim}_{T} S$. Then we have the following.

(1) Suppose that $s=d$. Then

(i) $\mathrm{e}_{1}(I)=\mathrm{e}_{0}(I)+\mathrm{e}_{1}(Q)-\ell_{A}(A / I)+\mathrm{e}_{0}(S)$ and

(ii) $\mathrm{e}_{i}(I)=\mathrm{e}_{i-1}(Q)+\mathrm{e}_{i}(Q)+\mathrm{e}_{i-1}(S)$ for all $2 \leq i \leq d$.

(2) Suppose that $s<d$. Then 
(i) $\mathrm{e}_{1}(I)=\mathrm{e}_{0}(I)+\mathrm{e}_{1}(Q)-\ell_{A}(A / I)$,

(ii) $\mathrm{e}_{i}(I)=\mathrm{e}_{i-1}(Q)+\mathrm{e}_{i}(Q)$ for $2 \leq i \leq d-s$, and

(iii) $\mathrm{e}_{i}(I)=\mathrm{e}_{i-1}(Q)+\mathrm{e}_{i}(Q)+(-1)^{d-s} \mathrm{e}_{i-d+s-1}(S)$ for all $d-s+1 \leq$ $i \leq d$.

We need the following lemma.

Lemma 2.6. Suppose that conditions $\left(\mathrm{C}_{1}\right)$ and $\left(\mathrm{C}_{2}\right)$ are satisfied. Then, for each $0 \leq i \leq d$, we have

$$
\left[\mathrm{H}_{M}^{i}(I T)\right]_{n} \cong \begin{cases}\mathrm{H}_{\mathfrak{m}}^{0}(A) & \text { if } i=n=0 \\ \mathrm{H}_{\mathfrak{m}}^{i-1}(A) & \text { if } 3 \leq i \leq d, 2-i \leq n \leq-1 \\ (0) & \text { otherwise }\end{cases}
$$

for all $n \in \mathbb{Z}$. Hence IT is a generalized Cohen-Macaulay T-module with $\operatorname{dim}_{T} I T=d+1$.

Recall that, once condition $\left(\mathrm{C}_{1}\right)$ is satisfied, all the local cohomology modules $\left\{\mathrm{H}_{\mathfrak{m}}^{i}(A)\right\}_{0 \leq i \leq d-1}$ of $A$ are finitely generated (see [21, Theorem 2.1]).

Proof of Lemma 2.6. Taking the local cohomology functor $\mathrm{H}_{M}^{i}(*)$ to the exact sequence

$$
0 \rightarrow I T \rightarrow T \rightarrow F \rightarrow 0
$$

we have isomorphisms $\mathrm{H}_{M}^{i}(I T) \cong \mathrm{H}_{M}^{i}(T)$ of graded $T$-modules for $0 \leq i \leq$ $d-1$, and the exact sequence

$$
0 \rightarrow \mathrm{H}_{M}^{d}(I T) \rightarrow \mathrm{H}_{M}^{d}(T) \rightarrow \mathrm{H}_{M}^{d}(F)
$$

of local cohomology modules, because $F \cong(A / I)\left[X_{1}, X_{2}, \ldots, X_{d}\right]$ is the polynomial ring over $A / I$ by Lemma 2.2 .

Then since $\left[\mathrm{H}_{M}^{d}(T)\right]_{n}=(0)$ for all $n \leq 1-d$ by [21, Theorem 6.2] and since $\left[\mathrm{H}_{M}^{d}(F)\right]_{n}=(0)$ for all $n \geq 1-d$, we have $\mathrm{H}_{M}^{d}(I T) \cong \mathrm{H}_{M}^{d}(T)$ as graded $T$ modules. Therefore, by [21, Theorem 6.2], we get the required assertion.

For a graded $T$-module $L$,

$$
\operatorname{g-depth}_{M} L:=\sup \left\{\ell \in \mathbb{Z} \mid M \subseteq \sqrt{\operatorname{Ann}_{T} \mathrm{H}_{M}^{i}(L)} \text { for all } i<\ell\right\}
$$

denotes the generalized depth of $L$ with respect to $M$ (see [12, Section 2]).

Then we have the following result which concerns a relationship between the generalized depth of Sally modules $S$ and associated graded rings $G$ of $I$. 
Proposition 2.7. Suppose that conditions $\left(\mathrm{C}_{1}\right),\left(\mathrm{C}_{2}\right)$, and $\left(\mathrm{C}_{3}\right)$ are satisfied. Assume that $S \neq(0)$, and put $s=\mathrm{g}-\operatorname{depth}_{M} S$. Then g-depth ${ }_{M} G=$ $s-1$ if $s<d$, and $S$ is a generalized Cohen-Macaulay $T$-module if and only if $\mathrm{g}$-depth ${ }_{M} G \geq d-1$.

Proof. Recall that $s \leq \operatorname{dim}_{T} S=d$ by Lemma 2.3. Because $I T$ is a generalized Cohen-Macaulay $T$-module with $\operatorname{dim}_{T} I T=d+1$ by Lemma 2.6 , it follows from the exact sequence

$$
\text { (a) } 0 \rightarrow I T \rightarrow I R \rightarrow S \rightarrow 0
$$

that $\mathrm{g}$-depth ${ }_{M} I R \geq d$ if $s=d$, and g-depth ${ }_{M} I R=s$ if $s<d$. Since $A$ is a generalized Cohen-Macaulay $\operatorname{ring}$ with $\operatorname{dim} A=d$, from the exact sequence

$$
\text { (b) } 0 \rightarrow I R(-1) \rightarrow R \rightarrow A \rightarrow 0
$$

it follows that g-depth ${ }_{M} R \geq d$ if $s=d$ and that g-depth ${ }_{M} R=$ g-depth ${ }_{M} I R$ if $s<d$. Therefore, the exact sequence

$$
\text { (c) } 0 \rightarrow I R \rightarrow R \rightarrow G \rightarrow 0
$$

implies that g-depth ${ }_{M} G \geq d-1$ if $s=d$. If $s<d$, then g-depth ${ }_{M} R=s$, and so by [12, Proposition 3.2] we get g-depth ${ }_{M} G=s-1$.

Suppose that g-depth ${ }_{M} G \geq d-1$. Then g-depth ${ }_{M} R \geq d$ by [12, Proposition 3.2], whence by the exact sequence (c) we have g-depth ${ }_{M} I R \geq d$, and so g-depth ${ }_{M} S \geq d$ by the exact sequence (a). Therefore, $S$ is a generalized Cohen-Macaulay $T$-module.

\section{$\S 3$. Preliminary steps for the proof}

In this section, we introduce some techniques, being inspired by [1] and [2], which play a crucial role throughout this paper. Let us begin with the following.

Lemma 3.1. Assume that $I \supsetneq Q$, and put $\mu=\mu_{A}(I / Q)$. Then there exists an exact sequence

$$
T(-1)^{\mu} \stackrel{\phi}{\rightarrow} R / T \rightarrow S(-1) \rightarrow 0
$$

as graded T-modules.

Proof. Let us write $I=Q+\left(x_{1}, x_{2}, \ldots, x_{\mu}\right)$, and let

$$
\phi: T(-1)^{\mu} \rightarrow R / T
$$


denote a homomorphism of graded $T$-modules with $\phi\left(\alpha_{1}, \alpha_{2}, \ldots, \alpha_{\mu}\right)=$ $\sum_{i=1}^{\mu} \overline{\alpha_{i} x_{i} t} \in R / T$ for $\alpha_{i} \in T$ and $1 \leq i \leq \mu$, where $\overline{\alpha_{i} x_{i} t}$ denotes the image of $\alpha_{i} x_{i} t$ in $R / T$. Then we have

$$
\text { Coker } \phi=R / I t \cdot T+T \cong R_{+} / I t \cdot T
$$

as graded $T$-modules. Then two isomorphisms

$$
R_{+} \stackrel{\widehat{t^{-1}}}{\rightarrow} I R(-1) \quad \text { and } \quad I t \cdot T \stackrel{\widehat{t^{-1}}}{\rightarrow} I T(-1)
$$

of graded $T$-modules induce the isomorphism $R_{+} / I t \cdot T \cong(I R / I T)(-1)$ of graded $T$-modules. Therefore, Coker $\phi \cong S(-1)$ as graded $T$-modules, whence we get a required exact sequence.

Tensoring the exact sequence of Lemma 3.1 with $A / I$, we get an exact sequence

$$
\text { (i) } 0 \rightarrow \operatorname{Ker} \bar{\phi} \rightarrow F(-1)^{\mu} \stackrel{\bar{\phi}}{\rightarrow} R / I R+T \rightarrow(S / I S)(-1) \rightarrow 0
$$

of graded $T$-modules, where $\bar{\phi}=A / I \otimes \phi$.

Furthermore, tensoring the exact sequence

$$
0 \rightarrow[\operatorname{Ker} \bar{\phi}]_{1} \rightarrow F_{0}^{\mu} \rightarrow[R / I R+T]_{1} \rightarrow 0
$$

with $F$, we get the following commutative diagram:

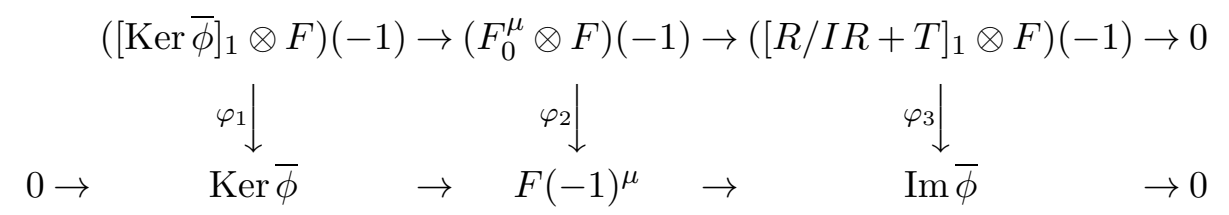

of graded $T$-modules. Then, since the map $\varphi_{2}$ is bijective, we have an exact sequence

$$
\text { (ii) } 0 \rightarrow K^{(1)} \rightarrow\left([R / I R+T]_{1} \otimes F\right)(-1) \stackrel{\varphi_{3}}{\rightarrow} \operatorname{Im} \bar{\phi} \rightarrow 0
$$

of graded $T$-modules, where $K^{(1)}=\operatorname{Ker} \varphi_{3}$.

We also have an exact sequence

$$
\text { (iii) } \quad 0 \rightarrow K^{(2)} \rightarrow F \stackrel{\bar{i}}{\rightarrow} G \rightarrow R / I R+T \rightarrow 0
$$


of graded $T$-modules induced by tensoring the canonical exact sequence

$$
0 \rightarrow T \stackrel{i}{\hookrightarrow} R \rightarrow R / T \rightarrow 0
$$

of graded $T$-modules with $A / I$, where $\bar{i}=A / I \otimes i$ and $K^{(2)}=\operatorname{Ker} \bar{i}$. Put $K^{(3)}=I S$.

Notice that $\operatorname{dim}_{T} K^{(i)} \leq d$ for all $1 \leq i \leq 3$, because $K^{(1)}$ and $K^{(2)}$ are embedded into $\left([R / I R+T]_{1} \otimes F\right)(-1)$ and $F$, respectively, and $\operatorname{dim}_{T} S \leq d$ by Remark 2.1 .

We then have the following inequality, which is proved by $[18$, Theorem 4.1] and [1, Theorem 3.1].

Proposition 3.2. We have

$$
2 \mathrm{e}_{0}(I)-\mathrm{e}_{1}(I)+\mathrm{e}_{1}(Q) \leq 2 \ell_{A}(A / I)+\ell_{A}\left(I / I^{2}+Q\right) .
$$

Proof. When $I=Q$, the required inequality is automatically satisfied. Therefore, we may assume that $I \supsetneq Q$. We then have

$$
\begin{aligned}
\ell_{A}\left([R / I R+T]_{n}\right) & =\ell_{A}\left([\operatorname{Im} \bar{\phi}]_{n}\right)+\ell_{A}\left([S / I S]_{n-1}\right), \\
\ell_{A}\left([\operatorname{Im} \bar{\phi}]_{n}\right) & =\ell_{A}\left(\left[[R / I R+T]_{1} \otimes F\right]_{n-1}\right)-\ell_{A}\left(\left[K^{(1)}\right]_{n}\right)
\end{aligned}
$$

for all $n \geq 0$ by the exact sequences (i) and (ii), and we have

$$
\ell_{A}\left([S / I S]_{n-1}\right)=\ell_{A}\left(S_{n-1}\right)-\ell_{A}\left(\left[K^{(3)}\right]_{n-1}\right)
$$

for all $n \geq 0$. Therefore, by the exact sequence (iii) we have

$$
\begin{aligned}
\ell_{A}\left(I^{n} / I^{n+1}\right)= & \ell_{A}\left(G_{n}\right)=\ell_{A}\left(F_{n}\right)+\ell_{A}\left([R / I R+T]_{n}\right)-\ell_{A}\left(\left[K^{(2)}\right]_{n}\right) \\
= & \ell_{A}\left(F_{n}\right)+\left\{\ell_{A}\left([\operatorname{Im} \bar{\phi}]_{n}\right)+\ell_{A}\left([S / I S]_{n-1}\right)\right\}-\ell_{A}\left(\left[K^{(2)}\right]_{n}\right) \\
= & \ell_{A}\left(F_{n}\right)+\left\{\ell_{A}\left(\left[[R / I R+T]_{1} \otimes F\right]_{n-1}\right)-\ell_{A}\left(\left[K^{(1)}\right]_{n}\right)\right\} \\
& +\left\{\ell_{A}\left(S_{n-1}\right)-\ell_{A}\left(\left[K^{(3)}\right]_{n-1}\right)\right\}-\ell_{A}\left(\left[K^{(2)}\right]_{n}\right)
\end{aligned}
$$

for all $n \geq 0$. Because $F$ is a homomorphic image of the polynomial ring $(A / I)\left[X_{1}, X_{2}, \ldots, X_{d}\right]$ over $A / I$, we have

$$
\ell_{A}\left(\left[[R / I R+T]_{1} \otimes F\right]_{n-1}\right) \leq \ell_{A}\left(I / I^{2}+Q\right)\left(\begin{array}{c}
n-1+d-1 \\
d-1
\end{array}\right)
$$


for all $n \geq 0$. Therefore, since $\operatorname{dim}_{T} K^{(i)} \leq d$ for all $1 \leq i \leq 3$, we have

$$
\begin{cases}\mathrm{e}_{0}(I) \leq \ell_{A}(A / I)+\ell_{A}\left(I / I^{2}+Q\right)+\mathrm{e}_{0}(S) & \text { if } \operatorname{dim}_{T} S=d \\ \mathrm{e}_{0}(I) \leq \ell_{A}(A / I)+\ell_{A}\left(I / I^{2}+Q\right) & \text { if } \operatorname{dim}_{T} S \leq d-1\end{cases}
$$

Hence, because $\mathrm{e}_{1}(I)-\mathrm{e}_{0}(I)-\mathrm{e}_{1}(Q)+\ell_{A}(A / I) \geq \mathrm{e}_{0}(S)$ if $\operatorname{dim}_{T} S=d$ by $[1$, Proposition 2.8] and [18, Corollary 6.1], and because $\mathrm{e}_{1}(I)-\mathrm{e}_{0}(I)-\mathrm{e}_{1}(Q)+$ $\ell_{A}(A / I) \geq 0$ by $[6$, Theorem 3.1], we have

$$
\mathrm{e}_{0}(I) \leq \ell_{A}(A / I)+\ell_{A}\left(I / I^{2}+Q\right)+\left\{\mathrm{e}_{1}(I)-\mathrm{e}_{0}(I)-\mathrm{e}_{1}(Q)+\ell_{A}(A / I)\right\} .
$$

Thus, we get the required inequality.

We then have the following.

Lemma 3.3. Suppose that conditions $\left(\mathrm{C}_{0}\right)$ and $\left(\mathrm{C}_{2}\right)$ are satisfied, and assume that $I \supsetneq Q$. Then we have

$$
\begin{aligned}
\ell_{A}\left(I^{n} / I^{n+1}\right)= & \left\{\ell_{A}(A / I)+\ell_{A}\left(I / I^{2}+Q\right)\right\}\left(\begin{array}{c}
n+d-1 \\
d-1
\end{array}\right) \\
& -\ell_{A}\left(I / I^{2}+Q\right)\left(\begin{array}{c}
n+d-2 \\
d-2
\end{array}\right) \\
& +\ell_{A}\left(S_{n-1}\right)-\ell_{A}\left(\left[K^{(1)}\right]_{n}\right)-\ell_{A}\left(\left[K^{(2)}\right]_{n}\right)-\ell_{A}\left(\left[K^{(3)}\right]_{n-1}\right)
\end{aligned}
$$

for all $n \geq 0$.

Proof. By the same argument as in the proof of Proposition 3.2, we have

$$
\begin{aligned}
\ell_{A}\left(I^{n} / I^{n+1}\right)= & \ell_{A}\left(F_{n}\right)+\ell_{A}\left(\left[[R / I R+T]_{1} \otimes F\right]_{n-1}\right)+\ell_{A}\left(S_{n-1}\right) \\
& -\ell_{A}\left(\left[K^{(1)}\right]_{n}\right)-\ell_{A}\left(\left[K^{(3)}\right]_{n-1}\right)-\ell_{A}\left(\left[K^{(2)}\right]_{n}\right)
\end{aligned}
$$

for all $n \geq 0$. Since conditions $\left(\mathrm{C}_{0}\right)$ and $\left(\mathrm{C}_{2}\right)$ are satisfied, $F \cong(A / I)\left[X_{1}, X_{2}\right.$, $\left.\ldots, X_{d}\right]$ is the polynomial ring over $A / I$ by Lemma 2.2 , whence $[R / I R+$ $T]_{1} \otimes F \cong\left(I / I^{2}+Q\right) \otimes(A / I)\left[X_{1}, X_{2}, \ldots, X_{d}\right]$. Therefore, we have

$$
\ell_{A}\left(F_{n}\right)=\ell_{A}(A / I)\left(\begin{array}{c}
n+d-1 \\
d-1
\end{array}\right)
$$

and

$$
\ell_{A}\left(\left[[R / I R+T]_{1} \otimes F\right]_{n-1}\right)=\ell_{A}\left(I / I^{2}+Q\right)\left(\begin{array}{c}
n+d-2 \\
d-1
\end{array}\right)
$$


for all $n \geq 0$. Hence, we have

$$
\begin{aligned}
\ell_{A}\left(I^{n} / I^{n+1}\right)= & \ell_{A}(A / I)\left(\begin{array}{c}
n+d-1 \\
d-1
\end{array}\right)+\ell_{A}\left(I / I^{2}+Q\right)\left(\begin{array}{c}
n+d-2 \\
d-1
\end{array}\right) \\
& +\ell_{A}\left(S_{n-1}\right)-\ell_{A}\left(\left[K^{(1)}\right]_{n}\right)-\ell_{A}\left(\left[K^{(3)}\right]_{n-1}\right)-\ell_{A}\left(\left[K^{(2)}\right]_{n}\right) \\
= & \ell_{A}(A / I)\left(\begin{array}{c}
n+d-1 \\
d-1
\end{array}\right) \\
& +\ell_{A}\left(I / I^{2}+Q\right)\left\{\left(\begin{array}{c}
n+d-1 \\
d-1
\end{array}\right)-\left(\begin{array}{c}
n+d-2 \\
d-2
\end{array}\right)\right\} \\
& +\ell_{A}\left(S_{n-1}\right)-\ell_{A}\left(\left[K^{(1)}\right]_{n}\right)-\ell_{A}\left(\left[K^{(3)}\right]_{n-1}\right)-\ell_{A}\left(\left[K^{(2)}\right]_{n}\right) \\
= & \left\{\ell_{A}(A / I)+\ell_{A}\left(I / I^{2}+Q\right)\right\}\left(\begin{array}{c}
n+d-1 \\
d-1
\end{array}\right) \\
& -\ell_{A}\left(I / I^{2}+Q\right)\left(\begin{array}{c}
n+d-2 \\
d-2
\end{array}\right) \\
& +\ell_{A}\left(S_{n-1}\right)-\ell_{A}\left(\left[K^{(1)}\right]_{n}\right)-\ell_{A}\left(\left[K^{(3)}\right]_{n-1}\right)-\ell_{A}\left(\left[K^{(2)}\right]_{n}\right)
\end{aligned}
$$

for all $n \geq 0$.

Recall that $\operatorname{dim}_{T} K^{(i)} \leq d$ for $1 \leq i \leq 3$, and put

$$
\Gamma=\left\{i \mid 1 \leq i \leq 3, \operatorname{dim}_{T} K^{(i)}=d\right\} .
$$

Then, by Lemma 3.3, we get the following result.

Lemma 3.4. Suppose that conditions $\left(\mathrm{C}_{0}\right)$ and $\left(\mathrm{C}_{2}\right)$ are satisfied, and assume that $I \supsetneq Q$. Then we have

$$
2 \mathrm{e}_{0}(I)-\mathrm{e}_{1}(I)+\mathrm{e}_{1}(Q)=2 \ell_{A}(A / I)+\ell_{A}\left(I / I^{2}+Q\right)-\sum_{i \in \Gamma} \mathrm{e}_{0}\left(K^{(i)}\right),
$$

where $\mathrm{e}_{0}\left(K^{(i)}\right)$ denotes the multiplicity of the graded $T$-module $K^{(i)}$ for $1 \leq$ $i \leq 3$.

Proof. By Lemma 3.3, we have

$$
\left\{\begin{array}{l}
\mathrm{e}_{0}(I)=\ell_{A}(A / I)+\ell_{A}\left(I / I^{2}+Q\right)+\mathrm{e}_{0}(S)-\sum_{i \in \Gamma} \mathrm{e}_{0}\left(K^{(i)}\right) \\
\quad \text { if } \operatorname{dim}_{T} S=d, \\
\mathrm{e}_{0}(I)=\ell_{A}(A / I)+\ell_{A}\left(I / I^{2}+Q\right)-\sum_{i \in \Gamma} \mathrm{e}_{0}\left(K^{(i)}\right) \\
\quad \text { if } \operatorname{dim}_{T} S \leq d-1 .
\end{array}\right.
$$


Meanwhile, since

$$
\begin{cases}\mathrm{e}_{1}(I)-\mathrm{e}_{0}(I)-\mathrm{e}_{1}(Q)+\ell_{A}(A / I)=\mathrm{e}_{0}(S) & \text { if } \operatorname{dim}_{T} S=d, \\ \mathrm{e}_{1}(I)-\mathrm{e}_{0}(I)-\mathrm{e}_{1}(Q)+\ell_{A}(A / I)=0 & \text { if } \operatorname{dim}_{T} S \leq d-1\end{cases}
$$

by Corollary 2.5 (see also [1, Theorem 2.8]), we have

$$
\begin{aligned}
\mathrm{e}_{0}(I)= & \ell_{A}(A / I)+\ell_{A}\left(I / I^{2}+Q\right) \\
& +\left\{\mathrm{e}_{1}(I)-\mathrm{e}_{0}(I)-\mathrm{e}_{1}(Q)+\ell_{A}(A / I)\right\}-\sum_{i \in \Gamma} \mathrm{e}_{0}\left(K^{(i)}\right) .
\end{aligned}
$$

Thus, we get the required equality.

In our proof of Theorem 1.1, we need the following.

Proposition 3.5. Suppose that conditions $\left(\mathrm{C}_{1}\right),\left(\mathrm{C}_{2}\right)$, and $\left(\mathrm{C}_{3}\right)$ are satisfied. Assume that $I \supsetneq Q$. Then the following three conditions are equivalent:

(1) we have $2 \mathrm{e}_{0}(I)-\mathrm{e}_{1}(I)+\mathrm{e}_{1}(Q)=2 \ell_{A}(A / I)+\ell_{A}\left(I / I^{2}+Q\right)$;

(2) there exist exact sequences

$$
0 \rightarrow\left(\left(I / I^{2}+Q\right) \otimes F\right)(-1) \rightarrow R / I R+T \rightarrow S(-1) \rightarrow 0
$$

and

$$
0 \rightarrow F \rightarrow G \rightarrow R / I R+T \rightarrow 0
$$

of graded $T$-modules; and

(3) $K^{(i)}=(0)$ for all $1 \leq i \leq 3$.

Proof. Because of the fact that conditions $\left(\mathrm{C}_{1}\right),\left(\mathrm{C}_{2}\right)$, and $\left(\mathrm{C}_{3}\right)$ are satisfied, $F \cong(A / I)\left[X_{1}, X_{2}, \ldots, X_{d}\right]$ is a polynomial ring over $A / I$ by Lemma 2.2 , whence $[R / I R+T]_{1} \otimes F \cong\left(I / I^{2}+Q\right) \otimes(A / I)\left[X_{1}, X_{2}, \ldots, X_{d}\right]$.

$(1) \Rightarrow(3)$ We have $\operatorname{Ass}_{T} K^{(1)} \subseteq\{\mathfrak{m} T\}$ by the exact sequence (ii), because $[R / I R+T]_{1} \otimes F$ is a maximal Cohen-Macaulay $F$-module. We also have $\operatorname{Ass}_{T} K^{(i)} \subseteq\{\mathfrak{m} T\}$ for all $2 \leq i \leq 3$ by the exact sequence (iii) and Lemma 2.3. Hence, $\operatorname{dim}_{T} K^{(i)}=d$ if $K^{(i)} \neq(0)$ for each $1 \leq i \leq 3$. Then we have

$$
\sum_{i \in \Gamma} \mathrm{e}_{0}\left(K^{(i)}\right)=\left\{2 \ell_{A}(A / I)+\ell_{A}\left(I / I^{2}+Q\right)\right\}-\left\{2 \mathrm{e}_{0}(I)-\mathrm{e}_{1}(I)+\mathrm{e}_{1}(Q)\right\}=0
$$

by Lemma 3.4. Therefore, $\operatorname{dim}_{T} K^{(i)}<d$, whence $K^{(i)}=(0)$ for all $1 \leq i \leq 3$.

$(3) \Rightarrow(2)$ This is obvious by the exact sequences (i), (ii), and (iii). 
$(2) \Rightarrow(1)$ By our assertion, we have

$$
\begin{aligned}
\ell_{A}\left(I^{n} / I^{n+1}\right) & =\ell_{A}\left(F_{n}\right)+\ell_{A}\left([R / I R+T]_{n}\right) \\
& =\ell_{A}\left(F_{n}\right)+\left\{\ell_{A}\left(\left[\left(I / I^{2}+Q\right) \otimes F\right]_{n-1}\right)+\ell_{A}\left(S_{n-1}\right)\right\}
\end{aligned}
$$

for all $n \geq 0$. Then, by the same argument as in the proof of Lemma 3.4, we get $2 \mathrm{e}_{0}(I)-\mathrm{e}_{1}(I)+\mathrm{e}_{1}(Q)=2 \ell_{A}(A / I)+\ell_{A}\left(I / I^{2}+Q\right)$, as required.

We get the following corollary by Proposition 3.5.

Corollary 3.6. Suppose that conditions $\left(\mathrm{C}_{1}\right),\left(\mathrm{C}_{2}\right)$, and $\left(\mathrm{C}_{3}\right)$ are satisfied. Assume that $2 \mathrm{e}_{0}(I)-\mathrm{e}_{1}(I)+\mathrm{e}_{1}(Q)=2 \ell_{A}(A / I)+\ell_{A}\left(I / I^{2}+Q\right)$. Then we have the following:

(1) $I^{n+2} \subseteq Q^{n} I$ for all $n \geq 0$,

(2) $Q^{n} \cap I^{n+1}=Q^{n} I$ for all $n \geq 0$,

(3) $\operatorname{depth} G>0$, and

(4) $\left(a_{1}, a_{2}, \ldots, \check{a_{i}}, \ldots, a_{d}\right): a_{i} \subseteq I^{2}+Q$ for all $1 \leq i \leq d$.

Proof. Suppose that $I=Q$. Then assertions (1) and (2) are naturally satisfied. Since $\ell_{A}(A / Q)=\mathrm{e}_{0}(Q)$ holds true, the base local ring $A$ is CohenMacaulay. Therefore, assertions (3) and (4) are also satisfied. Thus, we may assume that $I \supsetneq Q$.

(1) By Proposition 3.5, we see that $I S=K^{(3)}=(0)$. Hence, $I^{n+2} \subseteq Q^{n} I$ for all $n \geq 0$ by Remark 2.1 .

(2) We see that $K^{(2)}=(0)$ by Proposition 3.5. Therefore, because $\left[K^{(2)}\right]_{n} \cong$ $\left[Q^{n} \cap I^{n+1}\right] / Q^{n} I$ for $n \geq 0$, we have $Q^{n} \cap I^{n+1}=Q^{n} I$ for all $n \geq 0$.

(3) We have exact sequences

$$
0 \rightarrow F \rightarrow G \rightarrow R / I R+T \rightarrow 0
$$

and

$$
0 \rightarrow\left(\left(I / I^{2}+Q\right) \otimes F\right)(-1) \rightarrow R / I R+T \rightarrow S(-1) \rightarrow 0
$$

of graded $T$-modules by Proposition 3.5. Since conditions $\left(\mathrm{C}_{1}\right),\left(\mathrm{C}_{2}\right)$, and $\left(\mathrm{C}_{3}\right)$ are satisfied, we have $F \cong(A / I)\left[X_{1}, X_{2}, \ldots, X_{d}\right]$ by Lemma 2.2 , whence $\left(I / I^{2}+Q\right) \otimes F \cong\left(I / I^{2}+Q\right) \otimes(A / I)\left[X_{1}, X_{2}, \ldots, X_{d}\right]$, and $\operatorname{Ass}_{T} S \subseteq\{\mathfrak{m} T\}$ by Lemma 2.3. Thus, we have $\operatorname{depth} G>0$ by the depth lemma.

(4) Take $x \in\left(a_{1}, a_{2}, \ldots, \check{a_{i}}, \ldots, a_{d}\right): a_{i}$ for $1 \leq i \leq d$, and write $a_{i} x=$ $\sum_{1 \leq j \leq d, j \neq i} a_{j} x_{j}$ with $x_{j} \in A$ for $1 \leq j \leq d, j \neq i$. Then we have $x, x_{j} \in I$ 
for all $1 \leq j \leq d, j \neq i$ by our condition $\left(\mathrm{C}_{2}\right)$. Let us look at the monomorphism

$$
\left(\left(I / I^{2}+Q\right) \otimes F\right)(-1) \rightarrow R / I R+T
$$

given by the composition map with $\varphi_{3}:\left(\left(I / I^{2}+Q\right) \otimes F\right)(-1) \rightarrow \operatorname{Im} \bar{\phi}$ and the inclusion $\operatorname{Im} \bar{\phi} \rightarrow R / I R+T$; so for simplicity, we say $\varphi_{3}$. Put

$$
g=\bar{x} \otimes \overline{a_{i} t}-\sum_{1 \leq j \leq d, j \neq i} \overline{x_{j}} \otimes \overline{a_{j} t} \in\left(I / I^{2}+Q\right) \otimes F,
$$

where $\bar{x}, \overline{x_{j}}$ denote the images of $x, x_{j}$ in $I / I^{2}+Q$ and $\overline{a_{i} t}, \overline{a_{j} t}$ denote the images of $a_{i} t, a_{j} t$ in $F$, respectively. Then we have

$$
\varphi_{3}(g)=\overline{x a_{i} t^{2}}-\sum_{1 \leq j \leq d, j \neq i} \overline{x_{j} a_{j} t^{2}}=0
$$

because $a_{i} x=\sum_{1 \leq j \leq d, j \neq i} a_{j} x_{j}$. Therefore, $g=\bar{x} \otimes \overline{a_{i} t}-\sum_{1 \leq j \leq d, j \neq i} \overline{x_{j}} \otimes$ $\overline{a_{j} t}=0$ since $\varphi_{3}$ is monomorphism. Then because $\left(I / I^{2}+Q\right) \otimes F \cong\left(I / I^{2}+\right.$ $Q) \otimes(A / I)\left[\overline{a_{1} t}, \overline{a_{2} t}, \ldots, \overline{a_{d} t}\right]$, we get $\bar{x}=0$, whence $x \in I^{2}+Q$, as required.

\section{§4. Proof of the main theorem: Part 1}

The purpose of this section is to give a proof of the equivalence of conditions (1) and (2) in Theorem 1.1. Let us note the following.

Lemma 4.1. Suppose that $d \geq 2$. Let $a \in Q \backslash \mathfrak{m} Q$ be a superficial element with respect to $I$ and $Q$. Let $\bar{A}=A /(a)$, let $\bar{I}=I /(a)$, and let $\bar{Q}=$ $Q /(a)$. Then $2 \mathrm{e}_{0}(I)-\mathrm{e}_{1}(I)+\mathrm{e}_{1}(Q)=2 \ell_{A}(A / I)+\ell_{A}\left(I / I^{2}+Q\right)$ if and only if $2 \mathrm{e}_{0}(\bar{I})-\mathrm{e}_{1}(\bar{I})+\mathrm{e}_{1}(\bar{Q})=2 \ell_{A}(\bar{A} / \bar{I})+\ell_{A}\left(\bar{I} / \bar{I}^{2}+\bar{Q}\right)$.

Proof. We have $\mathrm{e}_{i}(I)=\mathrm{e}_{i}(\bar{I})$ and $\mathrm{e}_{i}(Q)=\mathrm{e}_{i}(\bar{Q})$ for $0 \leq i \leq d-2$, and we have $\mathrm{e}_{d-1}(I)=\mathrm{e}_{d-1}(\bar{I})+(-1)^{d} \ell_{A}\left((0):_{A} a\right)$ and $\mathrm{e}_{d-1}(Q)=\mathrm{e}_{d-1}(\bar{Q})+$ $(-1)^{d} \ell_{A}\left((0):_{A} a\right)$. Hence, $2 \mathrm{e}_{0}(I)-\mathrm{e}_{1}(I)+\mathrm{e}_{1}(Q)=2 \mathrm{e}_{0}(\bar{I})-\mathrm{e}_{1}(\bar{I})+\mathrm{e}_{1}(\bar{Q})$. Meanwhile, we have $2 \ell_{A}(A / I)+\ell_{A}\left(I / I^{2}+Q\right)=2 \ell_{A}(\bar{A} / \bar{I})+\ell_{A}\left(\bar{I} / \bar{I}^{2}+\bar{Q}\right)$. Therefore, we get the required equivalence.

Lemma 4.2. Put $C=A / W$. Then $2 \mathrm{e}_{0}(I)-\mathrm{e}_{1}(I)+\mathrm{e}_{1}(Q)=2 \ell_{A}(A / I)+$ $\ell_{A}\left(I / I^{2}+Q\right)$ if and only if $2 \mathrm{e}_{0}(I C)-\mathrm{e}_{1}(I C)+\mathrm{e}_{1}(Q C)=2 \ell_{A}(C / I C)+$ $\ell_{A}\left(I C / I^{2} C+Q C\right)$ and $I^{2}+Q \supseteq W$.

Proof. Since $\mathrm{e}_{i}(I)=\mathrm{e}_{i}(I C)$ and $\mathrm{e}_{i}(Q)=\mathrm{e}_{i}(Q C)$ for all $0 \leq i \leq d-1$, and $\mathrm{e}_{d}(I)=\mathrm{e}_{d}(I C)+(-1)^{d} \ell_{A}(W)$ and $\mathrm{e}_{d}(Q)=\mathrm{e}_{d}(Q C)+(-1)^{d} \ell_{A}(W)$, we have $2 \mathrm{e}_{0}(I)-\mathrm{e}_{1}(I)+\mathrm{e}_{1}(Q)=2 \mathrm{e}_{0}(I C)-\mathrm{e}_{1}(I C)+\mathrm{e}_{1}(Q C)$. 
Assume that $2 \mathrm{e}_{0}(I)-\mathrm{e}_{1}(I)+\mathrm{e}_{1}(Q)=2 \ell_{A}(A / I)+\ell_{A}\left(I / I^{2}+Q\right)$. Then, since $2 \mathrm{e}_{0}(I C)-\mathrm{e}_{1}(I C)+\mathrm{e}_{1}(Q C) \leq 2 \ell_{A}(C / I C)+\ell_{A}\left(I C / I^{2} C+Q C\right)$ by Proposition 3.2 , and $\ell_{A}(C / I C) \leq \ell_{A}(A / I)$ and $\ell_{A}\left(I C / I^{2} C+Q C\right) \leq$ $\ell_{A}\left(I / I^{2}+Q\right)$, we have

$$
\begin{aligned}
2 \mathrm{e}_{0}(I)-\mathrm{e}_{1}(I)+\mathrm{e}_{1}(Q) & =2 \mathrm{e}_{0}(I C)-\mathrm{e}_{1}(I C)+\mathrm{e}_{1}(Q C) \\
& \leq 2 \ell_{A}(C / I C)+\ell_{A}\left(I C / I^{2} C+Q C\right) \\
& \leq 2 \ell_{A}(A / I)+\ell_{A}\left(I / I^{2}+Q\right) \\
& =2 \mathrm{e}_{0}(I)-\mathrm{e}_{1}(I)+\mathrm{e}_{1}(Q) .
\end{aligned}
$$

Therefore, equalities

$$
2 \mathrm{e}_{0}(I C)-\mathrm{e}_{1}(I C)+\mathrm{e}_{1}(Q C)=2 \ell_{A}(C / I C)+\ell_{A}\left(I C / I^{2} C+Q C\right),
$$

$\ell_{A}(C / I C)=\ell_{A}(A / I)$, and $\ell_{A}\left(I C / I^{2} C+Q C\right)=\ell_{A}\left(I / I^{2}+Q\right)$ hold true. Then we have $I \supseteq W$ since $\ell_{A}(A / I+W)=\ell_{A}(C / I C)=\ell_{A}(A / I)$. Hence, because

$$
\ell_{A}\left(I / I^{2}+Q+W\right)=\ell_{A}\left(I C / I^{2} C+Q C\right)=\ell_{A}\left(I / I^{2}+Q\right),
$$

we get $I^{2}+Q \supseteq W$.

Suppose that $2 \mathrm{e}_{0}(I C)-\mathrm{e}_{1}(I C)+\mathrm{e}_{1}(Q C)=2 \ell_{A}(C / I C)+\ell_{A}\left(I C / I^{2} C+\right.$ $Q C)$ and $I^{2}+Q \supseteq W$. Then we have $\ell_{A}(A / I)=\ell_{A}(C / I C)$ and $\ell_{A}\left(I / I^{2}+\right.$ $Q)=\ell_{A}\left(I C / I^{2} C+Q C\right)$, so that

$$
\begin{aligned}
2 \mathrm{e}_{0}(I)-\mathrm{e}_{1}(I)+\mathrm{e}_{1}(Q) & =2 \mathrm{e}_{0}(I C)-\mathrm{e}_{1}(I C)+\mathrm{e}_{1}(Q C) \\
& =2 \ell_{A}(C / I C)+\ell_{A}\left(I C / I^{2} C+Q C\right) \\
& =2 \ell_{A}(A / I)+\ell_{A}\left(I / I^{2}+Q\right),
\end{aligned}
$$

as required.

We are now in a position to prove the implication $(2) \Rightarrow(1)$ of Theorem 1.1.

Proof of $(2) \Rightarrow(1)$ of Theorem 1.1. Put $C=A / W$. Then we have $I^{3} C=$ $Q I^{2} C$ and $Q^{n} C \cap I^{n+1} C=Q^{n} I C$ for all $n \geq 1$. Since condition $\left(\mathrm{C}_{1}\right)$ is satisfied, we have $\left(a_{1}, a_{2}, \ldots, \check{a_{i}}, \ldots, a_{d}\right) C: a_{i} \subseteq I^{2} C+Q C$ for all $1 \leq i \leq d$. Therefore, passing to the ring $C$, we may assume that condition $\left(\mathrm{C}_{3}\right)$ is satisfied by Lemma 4.2 , because $W \subseteq\left(a_{1}, a_{2}, \ldots, \check{a_{i}}, \ldots, a_{d}\right): a_{i} \subseteq I^{2}+Q$ for 
$1 \leq i \leq d$. Then since $I^{3}=Q I^{2}$ and $Q^{n} \cap I^{n+1}=Q^{n} I$ for all $n \geq 1$, we have $K^{(3)}=I S=(0)$ and $K^{(2)}=(0)$.

We have to show that $K^{(1)}=(0)$, and notice that $\left[K^{(1)}\right]_{n}=(0)$ for all $n \leq 1$. Assume that $K^{(1)} \neq(0)$. Let $n \geq 2$ as the least integer so that $\left[K^{(1)}\right]_{n} \neq$ $(0)$, and take $0 \neq g \in\left[K^{(1)}\right]_{n}=\left[\operatorname{Ker} \varphi_{3}\right]_{n}$. Put

$$
\begin{aligned}
& \Gamma^{\prime}=\left\{\left(\alpha_{1}, \alpha_{2}, \ldots, \alpha_{d}\right) \in \mathbb{Z}^{d} \mid \alpha_{1} \geq 1, \alpha_{i} \geq 0 \text { for } 2 \leq i \leq d \text { and } \sum_{i=1}^{d} \alpha_{i}=n-1\right\}, \\
& \Gamma^{\prime \prime}=\left\{\left(0, \beta_{2}, \ldots, \beta_{d}\right) \in \mathbb{Z}^{d} \mid \beta_{i} \geq 0 \text { for } 2 \leq i \leq d \text { and } \sum_{i=2}^{d} \beta_{i}=n-1\right\} .
\end{aligned}
$$

Then since

$$
\Gamma^{\prime} \cup \Gamma^{\prime \prime}=\left\{\left(\alpha_{1}, \alpha_{2}, \ldots, \alpha_{d}\right) \in \mathbb{Z}^{d} \mid \alpha_{i} \geq 0 \text { for } 1 \leq i \leq d \text { and } \sum_{i=1}^{d} \alpha_{i}=n-1\right\},
$$

we may write

$$
\begin{aligned}
g= & \sum_{\alpha \in \Gamma^{\prime} \cup \Gamma^{\prime \prime}} \overline{x_{\alpha}} \otimes \overline{\left(a_{1} t\right)^{\alpha_{1}}\left(a_{2} t\right)^{\alpha_{2}} \cdots\left(a_{d} t\right)^{\alpha_{d}}} \\
= & \sum_{\alpha \in \Gamma^{\prime}} \overline{x_{\alpha}} \otimes \overline{\left(a_{1} t\right)^{\alpha_{1}}\left(a_{2} t\right)^{\alpha_{2}} \cdots\left(a_{d} t\right)^{\alpha_{d}}}+\sum_{\beta \in \Gamma^{\prime \prime}} \overline{x_{\beta}} \otimes \overline{\left(a_{2} t\right)^{\beta_{2}} \cdots\left(a_{d} t\right)^{\beta_{d}}} \\
& \in\left(I / I^{2}+Q\right) \otimes F_{n-1},
\end{aligned}
$$

with $x_{\alpha}, x_{\beta} \in I$, where $\overline{x_{\alpha}}$ and $\overline{x_{\beta}}$ denote the images of $x_{\alpha}$ and $x_{\beta}$ in $I / I^{2}+$

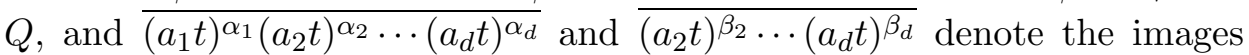
of $\left(a_{1} t\right)^{\alpha_{1}}\left(a_{2} t\right)^{\alpha_{2}} \cdots\left(a_{d} t\right)^{\alpha_{d}}$ and $\left(a_{2} t\right)^{\beta_{2}} \cdots\left(a_{d} t\right)^{\beta_{d}}$ in $F_{n-1}$, respectively. We then have

$$
\varphi_{3}(g)=\sum_{\alpha \in \Gamma^{\prime}} \overline{x_{\alpha} a_{1}^{\alpha_{1}} a_{2}^{\alpha_{2}} \cdots a_{d}^{\alpha_{d}} t^{n}}+\sum_{\beta \in \Gamma^{\prime \prime}} \overline{x_{\beta} a_{2}^{\beta_{2}} \cdots a_{d}^{\beta_{d}} t^{n}}=0,
$$

where $\overline{x_{\alpha} a_{1}^{\alpha_{1}} a_{2}^{\alpha_{2}} \cdots a_{d}^{\alpha_{d}} t^{n}}$ and $\overline{x_{\beta} a_{2}^{\beta_{2}} \cdots a_{d}^{\beta_{d}} t^{n}}$ denote the images of $x_{\alpha} a_{1}^{\alpha_{1}} a_{2}^{\alpha_{2}}$ $\cdots a_{d}^{\alpha_{d}} t^{n}$ and $x_{\beta} a_{2}^{\beta_{2}} \cdots a_{d}^{\beta_{d}} t^{n}$ in $[R / I R+T]_{n}$, respectively.

Then because

$$
\sum_{\alpha \in \Gamma^{\prime}} x_{\alpha} a_{1}^{\alpha_{1}} a_{2}^{\alpha_{2}} \cdots a_{d}^{\alpha_{d}}+\sum_{\beta \in \Gamma^{\prime \prime}} x_{\beta} a_{2}^{\beta_{2}} \cdots a_{d}^{\beta_{d}} \in I^{n+1}+Q^{n},
$$




$$
I^{n+1}+Q^{n}=Q^{n-1} I^{2}+Q^{n} \subseteq a_{1} Q^{n-2} I^{2}+a_{1} Q^{n-1}+\left(a_{2}, a_{3}, \ldots, a_{d}\right)^{n-1},
$$

and $\sum_{\beta \in \Gamma^{\prime \prime}} x_{\beta} a_{2}^{\beta_{2}} \cdots a_{d}^{\beta_{d}} \in\left(a_{2}, a_{3}, \ldots, a_{d}\right)^{n-1}$, there exist $q \in Q^{n-2} I^{2}$ and $q^{\prime} \in Q^{n-1}$ such that

$$
\begin{aligned}
a_{1} & \cdot\left\{\sum_{\alpha \in \Gamma^{\prime}} x_{\alpha} a_{1}^{\alpha_{1}-1} a_{2}^{\alpha_{2}} \cdots a_{d}^{\alpha_{d}}+q+q^{\prime}\right\} \\
& =\sum_{\alpha \in \Gamma^{\prime}} x_{\alpha} a_{1}^{\alpha_{1}} a_{2}^{\alpha_{2}} \cdots a_{d}^{\alpha_{d}}+a_{1} q+a_{1} q^{\prime} \in\left(a_{2}, a_{3}, \ldots, a_{d}\right)^{n-1} .
\end{aligned}
$$

Therefore, since $\left(a_{2}, a_{3}, \ldots, a_{d}\right)^{n-1}: a_{1} \subseteq\left(a_{2}, a_{3}, \ldots, a_{d}\right)^{n-2} \cdot\left(\left(a_{2}, a_{3}, \ldots, a_{d}\right)\right.$ : $\left.a_{1}\right)$ by $\left[8\right.$, Lemma 3.5] and $\left(a_{2}, a_{3}, \ldots, a_{d}\right): a_{1} \subseteq I^{2}+Q$, we have

$$
\sum_{\alpha \in \Gamma^{\prime}} x_{\alpha} a_{1}^{\alpha_{1}-1} a_{2}^{\alpha_{2}} \cdots a_{d}^{\alpha_{d}}+q+q^{\prime} \in\left(a_{2}, a_{3}, \ldots, a_{d}\right)^{n-2} \cdot\left(I^{2}+Q\right),
$$

and hence

$$
\sum_{\alpha \in \Gamma^{\prime}} x_{\alpha} a_{1}^{\alpha_{1}-1} a_{2}^{\alpha_{2}} \cdots a_{d}^{\alpha_{d}} \in I^{n}+Q^{n-1}
$$

by the choices of $q, q^{\prime}$. Hence, we get $\sum_{\alpha \in \Gamma^{\prime}} \overline{x_{\alpha}} \otimes \overline{\left(a_{1} t\right)^{\alpha_{1}-1}\left(a_{2} t\right)^{\alpha_{2}} \cdots\left(a_{d} t\right)^{\alpha_{d}}} \in$ $\left[K^{(1)}\right]_{n-1}=(0)$, whence

$$
g=\sum_{\beta \in \Gamma^{\prime \prime}} \overline{x_{\beta}} \otimes \overline{\left(a_{2} t\right)^{\beta_{2}} \cdots\left(a_{d} t\right)^{\beta_{d}}} .
$$

Therefore, $g=0$ by the symmetry among the elements $a_{i} t$, because, for all $1 \leq i \leq d$, we have $I^{n+1}+Q^{n}=Q^{n-1} I^{2}+Q^{n} \subseteq a_{i} Q^{n-2} I^{2}+a_{i} Q^{n-1}+$ $\left(a_{1}, a_{2}, \ldots, \check{a_{i}}, \ldots, a_{d}\right)^{n-1},\left(a_{1}, a_{2}, \ldots, \check{a_{i}}, \ldots, a_{d}\right)^{n-1}: a_{i} \subseteq\left(a_{1}, a_{2}, \ldots, \check{a_{i}}, \ldots\right.$, $\left.a_{d}\right)^{n-2} \cdot\left(\left(a_{1}, a_{2}, \ldots, \check{a_{i}}, \ldots, a_{d}\right): a_{i}\right)$ by $[8$, Lemma 3.5$]$, and $\left(a_{1}, a_{2}, \ldots, \check{a_{i}}, \ldots\right.$, $\left.a_{d}\right): a_{i} \subseteq I^{2}+Q$. But this is a contradiction. Thus, we get $K^{(1)}=(0)$ as required. Consequently, the required equality $2 \mathrm{e}_{0}(I)-\mathrm{e}_{1}(I)=2 \ell_{A}(A / I)+$ $\ell_{A}\left(I / I^{2}+Q\right)$ holds true by Proposition 3.5.

In the rest of Section 4 , we must show the implication $(1) \Rightarrow(2)$ of Theorem 1.1. Suppose that conditions $\left(\mathrm{C}_{1}\right)$ and $\left(\mathrm{C}_{2}\right)$ are satisfied, and assume that $2 \mathrm{e}_{0}(I)-\mathrm{e}_{1}(I)+\mathrm{e}_{1}(Q)=2 \ell_{A}(A / I)+\ell_{A}\left(I / I^{2}+Q\right)$. Put $C=A / W$; then we have $2 \mathrm{e}_{0}(I C)-\mathrm{e}_{1}(I C)+\mathrm{e}_{1}(Q C)=2 \ell_{A}(C / I C)+\ell_{A}\left(I C / I^{2} C+Q C\right)$ and $W \subseteq I^{2}+Q$ by Lemma 4.2. Therefore, passing to the $\operatorname{ring} C$, we get $\left(Q^{n}+W\right) \cap\left(I^{n+1}+W\right)=Q^{n} I+W$ for all $n \geq 1$ and $\left(a_{1}, a_{2}, \ldots, \check{a_{i}}, \ldots, a_{d}\right):$ $a_{i} \subseteq I^{2}+Q$ for all $1 \leq i \leq d$ by Corollary 3.6. Thus, the implication $(1) \Rightarrow(2)$ in Theorem 1.1 has been proven modulo the following Theorem 4.3. 
THEOREM 4.3. Suppose that conditions $\left(\mathrm{C}_{1}\right)$ and $\left(\mathrm{C}_{2}\right)$ are satisfied, and assume that $2 \mathrm{e}_{0}(I)-\mathrm{e}_{1}(I)+\mathrm{e}_{1}(Q)=2 \ell_{A}(A / I)+\ell_{A}\left(I / I^{2}+Q\right)$. Then we have $I^{3} \subseteq Q I^{2}+W$ and $I^{2} \supseteq W$.

Proof. We put $C=A / W$; then all conditions $\left(\mathrm{C}_{1}\right),\left(\mathrm{C}_{2}\right)$, and $\left(\mathrm{C}_{3}\right)$ are satisfied for the ring $C$ and the ideals $I C$ and $Q C$. We then have $2 \mathrm{e}_{0}(I C)-$ $\mathrm{e}_{1}(I C)+\mathrm{e}_{1}(Q C)=2 \ell_{A}(C / I C)+\ell_{A}\left(I C / I^{2}+Q C\right)$ and $I^{2}+Q \supseteq W$ by Lemma 4.2 .

Take $w \in W \subseteq I^{2}+Q$, and write $w=i+q$ with $i \in I^{2}$ and $q \in Q$. Then we have $\bar{q}=\overline{-i} \in Q C \cap I^{2} C=Q I C$ by Corollary 3.6(2), where $\bar{q}$ and $\overline{-i}$ denote the images of $q$ and $-i$ in $C$, respectively. Hence,

$$
q \in Q I+W \cap Q=Q I
$$

because $W \cap Q=(0)$ by condition $\left(\mathrm{C}_{1}\right)$ (see [21, Corollary 2.3]). Therefore, $w=i+q \in I^{2}$, whence $W \subseteq I^{2}$.

In the rest of our proof of Theorem 4.3, we have to show that $I^{3} \subseteq$ $Q I^{2}+W$. Passing to the $\operatorname{ring} C$, we may assume that all conditions $\left(\mathrm{C}_{1}\right)$, $\left(\mathrm{C}_{2}\right)$, and $\left(\mathrm{C}_{3}\right)$ are satisfied. Put $f_{1}=a_{1} t \in R$. Then, since $\operatorname{depth} G>0$ by Corollary 3.6(3), we may assume that $f_{1}$ is a regular element of $G$.

We proceed by induction on $d$. Suppose that $d=1$. Since $I^{3} \subseteq Q I \subseteq\left(a_{1}\right)$ by Corollary 3.6(1) and $f_{1}=a_{1}$ is $G$-regular, we have $I^{3}=I^{3} \cap\left(a_{1}\right)=a_{1} I^{2}$.

We may assume that $d \geq 2$ and that our assertion holds true for $d-1$. We put $\bar{A}=A /\left(a_{1}\right), \bar{I}=I \bar{A}$, and $\bar{Q}=Q \bar{A}$, and notice that conditions $\left(\mathrm{C}_{1}\right)$ and $\left(\mathrm{C}_{2}\right)$ are satisfied for the ring $\bar{A}$ and the ideals $\bar{I}$ and $\bar{Q}$. Then we have $2 \mathrm{e}_{0}(\bar{I})-\mathrm{e}_{1}(\bar{I})+\mathrm{e}_{1}(\bar{Q})=2 \ell_{A}(\bar{A} / \bar{I})+\ell_{A}\left(\bar{I} / \bar{I}^{2}+\bar{Q}\right)$ by Lemma 4.1. Hence, the hypothesis of induction on $d$ says that

$$
\bar{I}^{3} \subseteq \overline{Q I}^{2}+\mathrm{H}_{\mathfrak{m}}^{0}(\bar{A})
$$

holds true. Therefore, we have

$$
I^{3} \subseteq Q I^{2}+\left[\left(a_{1}\right): Q\right]
$$

because $a_{1}, a_{2}, \ldots, a_{d}$ forms a $d$-sequence on $A$. Take $x \in I^{3} \subseteq Q I^{2}+\left[\left(a_{1}\right)\right.$ : $Q]$, and write $x=y+z$ with $y \in Q I^{2}$ and $z \in\left[\left(a_{1}\right): Q\right]$. Then we have

$$
z=x-y \in\left[\left(a_{1}\right): Q\right] \cap I^{3} \subseteq\left[\left(a_{1}\right): Q\right] \cap Q=\left(a_{1}\right),
$$

because $I^{3} \subseteq Q I$ by Corollary 3.6(1) and $a_{1}, a_{2}, \ldots, a_{d}$ forms a $d$-sequence on $A$. Therefore, since $f_{1}=a_{1} t$ is a $G$-regular element, we have $z \in\left(a_{1}\right) \cap$ 
$I^{3}=a_{1} I^{2}$. Thus, we get $x=y+z \in Q I^{2}$, whence $I^{3}=Q I^{2}$. This completes the proof of Theorem 4.3 and proof of the equivalence of Theorem 1.1, conditions (1) and (2), as well.

\section{$\S 5$. Proof of the main theorem: Part 2}

The purpose of this section is to prove the last assertions in Theorem 1.1. To begin with, we note the following.

REmark 5.1. Assume that $I^{2} \subseteq Q$ and that $2 \mathrm{e}_{0}(I)-\mathrm{e}_{1}(I)+\mathrm{e}_{1}(Q)=$ $2 \ell_{A}(A / I)+\ell_{A}\left(I / I^{2}+Q\right)$. Then $A$ is a Cohen-Macaulay ring and $I^{2}=Q I$, so $S=(0)$ by Remark 2.1. Hence, $G$ is a Cohen-Macaulay ring, and the a-invariant a $(G)$ of $G$ is at most $1-d$.

Proof. Since $I^{2} \subseteq Q$, we have $\ell_{A}\left(I / I^{2}+Q\right)=\ell_{A}(I / Q)$. We then have

$$
\begin{aligned}
&\left\{2 \ell_{A}(A / I)+\ell_{A}\left(I / I^{2}+Q\right)\right\}-\left\{2 \mathrm{e}_{0}(I)-\mathrm{e}_{1}(I)+\mathrm{e}_{1}(Q)\right\} \\
&=\left\{2 \ell_{A}(A / I)+\ell_{A}(I / Q)\right\}-2 \mathrm{e}_{0}(I)+\mathrm{e}_{1}(I)-\mathrm{e}_{1}(Q) \\
& \geq \ell_{A}(A / I)+\ell_{A}(A / Q)-2 \mathrm{e}_{0}(I) \\
&+\left\{\mathrm{e}_{0}(I)+\mathrm{e}_{1}(Q)-\ell_{A}(A / I)\right\}-\mathrm{e}_{1}(Q) \\
&= \ell_{A}(A / Q)-\mathrm{e}_{0}(I)=\ell_{A}(A / Q)-\mathrm{e}_{0}(Q) \geq 0
\end{aligned}
$$

because $\mathrm{e}_{1}(I) \geq \mathrm{e}_{0}(I)+\mathrm{e}_{1}(Q)-\ell_{A}(A / I)$ by [6, Theorem 3.1]. Therefore, we have $\ell_{A}(A / Q)=\mathrm{e}_{0}(Q)$, whence the base local $\operatorname{ring} A$ is Cohen-Macaulay. Then we have $I^{2}=Q \cap I^{2}=Q I$ by Corollary 3.6(2). Thus, $G$ is a CohenMacaulay ring with $\mathrm{a}(G) \leq 1-d$.

We divide the proof of the last assertions in Theorem 1.1 into a few steps. Let us begin with the following.

Theorem 5.2. Suppose that conditions $\left(\mathrm{C}_{1}\right)$ and $\left(\mathrm{C}_{2}\right)$ are satisfied, and assume that $2 \mathrm{e}_{0}(I)-\mathrm{e}_{1}(I)+\mathrm{e}_{1}(Q)=2 \ell_{A}(A / I)+\ell_{A}\left(I / I^{2}+Q\right)$. Then $G$ is a generalized Cohen-Macaulay ring, and $S$ is a generalized Cohen-Macaulay T-module.

Proof. When $I^{2} \subseteq Q$, then $G$ is a Cohen-Macaulay ring and $S=(0)$ by Remark 5.1. Therefore, we may assume that $I^{2} \nsubseteq Q$; hence $S \neq(0)$. We put $C=A / W$. Then all conditions $\left(\mathrm{C}_{1}\right),\left(\mathrm{C}_{2}\right)$, and $\left(\mathrm{C}_{3}\right)$ are satisfied for the ring $C$ and the ideals $I C$ and $Q C$. We have $2 \mathrm{e}_{0}(I C)-\mathrm{e}_{1}(I C)+$ 
$\mathrm{e}_{1}(Q C)=2 \ell_{A}(C / I C)+\ell_{A}\left(I C / I^{2} C+Q C\right)$ by Lemma 4.2. Let us consider the canonical epimorphism

$$
G \stackrel{\varphi}{\longrightarrow} \mathrm{G}(I C)
$$

of graded rings. Then, since depth $\mathrm{G}(I C)>0$ by Corollary 3.6, it is easy to see that $\operatorname{Ker} \varphi \cong \mathrm{H}_{M}^{0}(G)$. Hence, we have $\mathrm{H}_{M}^{i}(G) \cong \mathrm{H}_{M}^{i}(\mathrm{G}(I C))$ as graded $T$-modules for all $i \geq 1$. Thus, passing to the $\operatorname{ring} C$, we may assume that condition $\left(\mathrm{C}_{3}\right)$ is satisfied.

By Proposition 3.5, we have exact sequences

$$
\begin{array}{ll}
\left(\dagger_{1}\right) \quad 0 \rightarrow\left(\left(I / I^{2}+Q\right) \otimes F\right)(-1) \rightarrow R / I R+T \rightarrow S(-1) \rightarrow 0 \\
\left(\dagger_{2}\right) \quad 0 \rightarrow F \rightarrow G \rightarrow R / I R+T \rightarrow 0
\end{array}
$$

of graded $T$-modules. Applying the local cohomology functor $\mathrm{H}_{M}^{i}(*)$ to the exact sequences $\left(\dagger_{1}\right)$ and $\left(\dagger_{2}\right)$, we get monomorphisms

$$
\mathrm{H}_{M}^{i}(G) \hookrightarrow \mathrm{H}_{M}^{i}(R / I R+T) \hookrightarrow \mathrm{H}_{M}^{i}(S)(-1)
$$

of local cohomology modules for $0 \leq i \leq d-1$ because $F \cong(A / I)\left[X_{1}, X_{2}\right.$, $\left.\ldots, X_{d}\right]$ is a polynomial ring over $A / I$ by Lemma 2.2 , whence $\left(I / I^{2}+Q\right) \otimes F$ is a maximal Cohen-Macaulay $F$-module. Then we have $s \leq$ g-depth ${ }_{M} G$, where $s=$ g-depth $M$. Therefore, $s=$ g-depth $M=d$ by Proposition 2.7.

The following Proposition 5.3 will give proofs of Theorem 1.1, assertions (i), (ii), and (iii).

Proposition 5.3. Suppose that conditions $\left(\mathrm{C}_{1}\right)$ and $\left(\mathrm{C}_{2}\right)$ are satisfied, and assume that $2 \mathrm{e}_{0}(I)-\mathrm{e}_{1}(I)+\mathrm{e}_{1}(Q)=2 \ell_{A}(A / I)+\ell_{A}\left(I / I^{2}+Q\right)$. Then we have the following.

(1) For all $n \in \mathbb{Z}$,

$$
\left[\mathrm{H}_{M}^{0}(G)\right]_{n} \cong \begin{cases}W / I^{3} \cap W & \text { if } n=2 \\ I^{n} \cap W / I^{n+1} \cap W & \text { if } n \geq 3 \\ (0) & \text { otherwise. }\end{cases}
$$

(2) We have $\mathrm{H}_{M}^{i}(G)=\left[\mathrm{H}_{M}^{i}(G)\right]_{2-i} \cong \mathrm{H}_{\mathfrak{m}}^{i}(A)$ for $1 \leq i \leq d-1$.

(3) We have a $(G) \leq 2-d$. 
Proof. When $I^{2} \subseteq Q$, then $G$ is a Cohen-Macaulay ring with a $(G) \leq 1-d$ by Remark 5.1. Hence, we may assume that $I^{2} \nsubseteq Q$, whence $S \neq(0)$. Let $C=A / W$. Then all conditions $\left(\mathrm{C}_{1}\right),\left(\mathrm{C}_{2}\right)$, and $\left(\mathrm{C}_{3}\right)$ are satisfied for the ring $C$ and the ideals $I C$ and $Q C$. We have $2 \mathrm{e}_{0}(I C)-\mathrm{e}_{1}(I C)+\mathrm{e}_{1}(Q C)=$ $2 \ell_{A}(C / I C)+\ell_{A}\left(I C / I^{2} C+Q C\right)$ by Lemma 4.2 .

Condition (1). Let us consider the canonical epimorphism

$$
G \stackrel{\varphi}{\longrightarrow} \mathrm{G}(I C)
$$

of graded rings. Then, for each $n \in \mathbb{Z}$, we have

$$
[\operatorname{Ker} \varphi]_{n} \cong I^{n} \cap W / I^{n+1} \cap W
$$

so that $\operatorname{Ker} \varphi$ is a finitely graded module because $I^{n} \cap W=(0)$ for all $n \gg 0$. Therefore, $\mathrm{H}_{M}^{0}(G)=\operatorname{Ker} \varphi$ since depth $\mathrm{G}(I C)>0$. Then because $W \subseteq I^{2}$ by Theorem 4.3, we have $\left[\mathrm{H}_{M}^{0}(G)\right]_{n}=(0)$ for $n=0,1$ and $\left[\mathrm{H}_{M}^{0}(G)\right]_{2}=W / I^{3} \cap W$.

Conditions (2) and (3). We proceed by induction on $d$. Passing to the ring $C$, we may assume that condition $\left(\mathrm{C}_{3}\right)$ is satisfied. Then, since $\operatorname{depth} G>0$ by Corollary 3.6(3), we may take $f_{1}=a_{1} t$ as $G$-regular.

Suppose that $d=1$; then $I^{3}=a_{1} I^{2}$ by Theorem 4.3 . Take the exact sequence

$$
0 \rightarrow G / f_{1} G \rightarrow \mathrm{H}_{M}^{1}(G)(-1) \stackrel{f_{1}}{\rightarrow} \mathrm{H}_{M}^{1}(G) \rightarrow \mathrm{H}_{M}^{1}(G / f G)
$$

of local cohomology modules. Then we have $\mathrm{a}_{1}(G) \leq 1$ because $G / f_{1} G \cong$ $A / I \oplus I /\left(I^{2}+Q\right) \oplus I^{2} / Q I$.

Assume that $d \geq 2$ and that our assertion holds true for $d-1$. We put $\bar{A}=$ $A /\left(a_{1}\right), \bar{I}=I \bar{A}$, and $\bar{Q}=Q \bar{A}$; then conditions $\left(\mathrm{C}_{1}\right)$ and $\left(\mathrm{C}_{2}\right)$ are satisfied for the ring $\bar{A}$ and the ideals $\bar{I}$ and $\bar{Q}$. Then we have $2 \mathrm{e}_{0}(\bar{I})-\mathrm{e}_{1}(\bar{I})+\mathrm{e}_{1}(\bar{Q})=$ $2 \ell_{A}(\bar{A} / \bar{I})+\ell_{A}\left(\bar{I} / \bar{I}^{2}+\bar{Q}\right)$ by Lemma 4.1 . By the hypothesis of induction on $d$, we get

$$
\mathrm{a}_{d-1}(\mathrm{G}(\bar{I})):=\max \left\{n \in \mathbb{Z} \mid\left[\mathrm{H}_{M}^{d-1}(\mathrm{G}(\bar{I}))\right]_{n} \neq(0)\right\} \leq 2-(d-1)=3-d
$$

and

$$
\mathrm{H}_{M}^{i}(\mathrm{G}(\bar{I}))=\left[\mathrm{H}_{M}^{i}(\mathrm{G}(\bar{I}))\right]_{2-i} \cong \mathrm{H}_{\mathfrak{m}}^{i}(\bar{A})
$$

for all $1 \leq i \leq d-2$. Since $I^{3}=Q I^{2}$ by Theorem 4.3 and condition $\left(\mathrm{C}_{1}\right)$ is satisfied, we have $\bar{I}^{n} \cap \mathrm{H}_{\mathfrak{m}}^{0}(\bar{A}) \subseteq \bar{Q} \cap \mathrm{H}_{\mathfrak{m}}^{0}(\bar{A})=(0)$ for all $n \geq 3$ by [21, Corollary 2.3]. Therefore, we have 


$$
\mathrm{H}_{M}^{0}(\mathrm{G}(\bar{I}))=\left[\mathrm{H}_{M}^{0}(\mathrm{G}(\bar{I}))\right]_{2} \cong \mathrm{H}_{\mathfrak{m}}^{0}(\bar{A})
$$

by assertion (1).

Because $f_{1}=a_{1} t$ forms $G$-regular element, we have $G / f_{1} G \cong \mathrm{G}(\bar{I})$. Apply local cohomology functors $\mathrm{H}_{M}^{i}(*)$ to the canonical exact sequences

$$
0 \rightarrow G(-1) \stackrel{f_{1}}{\rightarrow} G \rightarrow \mathrm{G}(\bar{I}) \rightarrow 0
$$

of graded rings and look at the derived long exact sequence

$$
\begin{aligned}
0 & \rightarrow \mathrm{H}_{M}^{0}(\mathrm{G}(\bar{I})) \rightarrow \mathrm{H}_{M}^{1}(G)(-1) \stackrel{f_{1}}{\rightarrow} \mathrm{H}_{M}^{1}(G) \rightarrow \mathrm{H}_{M}^{1}(\mathrm{G}(\bar{I})) \rightarrow \cdots \\
\cdots & \rightarrow \mathrm{H}_{M}^{i-1}(\mathrm{G}(\bar{I})) \rightarrow \mathrm{H}_{M}^{i}(G)(-1) \stackrel{f_{1}}{\rightarrow} \mathrm{H}_{M}^{i}(G) \rightarrow \mathrm{H}_{M}^{i}(\mathrm{G}(\bar{I})) \rightarrow \cdots \\
\cdots & \rightarrow \mathrm{H}_{M}^{d-1}(\mathrm{G}(\bar{I})) \rightarrow \mathrm{H}_{M}^{d}(G)(-1) \stackrel{f_{1}}{\rightarrow} \mathrm{H}_{M}^{d}(G) \rightarrow 0
\end{aligned}
$$

of local cohomology modules.

Setting $a=\mathrm{a}(G)$ in the exact sequence $(q)$ shows that

$$
\left[\mathrm{H}_{M}^{d}(G)(-1)\right]_{a+1}=\left[\mathrm{H}_{M}^{d}(G)\right]_{a}(\neq(0))
$$

is a homomorphic image of $\left[\mathrm{H}_{M}^{d-1}(\mathrm{G}(\bar{I}))\right]_{a+1}$. Hence, $a+1 \leq \mathrm{a}(\mathrm{G}(\bar{I})) \leq 3-d$, whence $a \leq 2-d$.

Because $\mathrm{H}_{M}^{i-1}(\mathrm{G}(\bar{I}))=\left[\mathrm{H}_{M}^{i-1}(\mathrm{G}(\bar{I}))\right]_{3-i}$ for all $1 \leq i \leq d-1$, we get monomorphisms

$$
0 \rightarrow\left[\mathrm{H}_{M}^{i}(G)\right]_{n-1} \rightarrow\left[\mathrm{H}_{M}^{i}(G)\right]_{n}
$$

for all $n \geq 4-i$ by the exact sequence $(\downarrow)$, whence $\left[\mathrm{H}_{M}^{i}(G)\right]_{n}=(0)$ for all $n \geq 3-i$.

On the other hand, since $\mathrm{H}_{M}^{i}(\mathrm{G}(\bar{I}))=\left[\mathrm{H}_{M}^{i}(\mathrm{G}(\bar{I}))\right]_{2-i}$ for all $1 \leq i \leq d-2$, we get epimorphisms

$$
\left[\mathrm{H}_{M}^{i}(G)\right]_{n-1} \rightarrow\left[\mathrm{H}_{M}^{i}(G)\right]_{n} \rightarrow 0
$$

for all $n \leq 1-i$ by the exact sequence $(\natural)$. Therefore, since $G$ is a generalized Cohen-Macaulay ring by Theorem 5.2, we have $\left[\mathrm{H}_{M}^{i}(G)\right]_{n}=(0)$ for all $1 \leq$ $i \leq d-2$ and $n \leq 1-i$. Thus, $\mathrm{H}_{M}^{i}(G)=\left[\mathrm{H}_{M}^{i}(G)\right]_{2-i}$ for all $1 \leq i \leq d-2$.

Then because

$$
0 \rightarrow\left[\mathrm{H}_{M}^{d-1}(G)\right]_{n-1} \rightarrow\left[\mathrm{H}_{M}^{d-1}(G)\right]_{n}
$$

for all $n \leq 2-d$, to prove $\mathrm{H}_{M}^{d-1}(G)=\left[\mathrm{H}_{M}^{d-1}(G)\right]_{3-d}$ it is enough to show that $\left[\mathrm{H}_{M}^{d-1}(G)\right]_{2-d}=(0)$. 
Thanks to Proposition 3.5, we have exact sequences

$$
\begin{array}{ll}
\left(\dagger_{1}\right) \quad 0 \rightarrow\left(\left(I / I^{2}+Q\right) \otimes F\right)(-1) \rightarrow R / I R+T \rightarrow S(-1) \rightarrow 0, \\
\left(\dagger_{2}\right) \quad 0 \rightarrow F \rightarrow G \rightarrow R / I R+T \rightarrow 0
\end{array}
$$

of graded $T$-modules so that applying local cohomology functors $\mathrm{H}_{M}^{i}(*)$, we get monomorphisms

$$
\left[\mathrm{H}_{M}^{d-1}(G)\right]_{2-d} \hookrightarrow\left[\mathrm{H}_{M}^{d-1}(R / I R+T)\right]_{2-d} \hookrightarrow\left[\mathrm{H}_{M}^{d-1}(S)\right]_{1-d}
$$

because $F \cong(A / I)\left[X_{1}, X_{2}, \ldots, X_{d}\right]$ is the polynomial ring over $A / I$ by Lemma 2.2, whence $\left(I / I^{2}+Q\right) \otimes F$ is a maximal Cohen-Macaulay $F$ module.

We now need the following claim.

Claim 1. For each $1 \leq i \leq d-1$, we have $\left[\mathrm{H}_{M}^{i}(I R)\right]_{n}=(0)$ for all $n \leq 2-i$ and $n<0$.

Proof. Let $1 \leq i \leq d-1$. Look at the exact sequence

$$
\mathrm{H}_{M}^{i-1}(G) \rightarrow \mathrm{H}_{M}^{i}(I R) \rightarrow \mathrm{H}_{M}^{i}(R)
$$

of local cohomology modules which is induced by the exact sequence

$$
0 \rightarrow I R \rightarrow R \rightarrow G \rightarrow 0
$$

of graded $T$-modules. Then, since $\mathrm{H}_{M}^{i-1}(G)=\left[\mathrm{H}_{M}^{i-1}(G)\right]_{3-i}$ by the above argument, we have monomorphisms

$$
\left[\mathrm{H}_{M}^{i}(I R)\right]_{n} \hookrightarrow\left[\mathrm{H}_{M}^{i}(R)\right]_{n}
$$

for all $n \leq 2-i$. We also have isomorphisms

$$
\left[\mathrm{H}_{M}^{i}(R)\right]_{n} \cong\left[\mathrm{H}_{M}^{i}(I R)\right]_{n-1}
$$

for all $n<0$, which are induced by the exact sequence

$$
0 \rightarrow I R(-1) \rightarrow R \rightarrow A \rightarrow 0
$$

of graded $T$-modules so that

$$
\left[\mathrm{H}_{M}^{i}(I R)\right]_{n} \hookrightarrow\left[\mathrm{H}_{M}^{i}(R)\right]_{n} \cong\left[\mathrm{H}_{M}^{i}(I R)\right]_{n-1}
$$

for all $n \leq 2-i$ and $n<0$. 
Therefore, since $\mathrm{H}_{M}^{i}(R)$ is finitely graded (because $G$ is a generalized Cohen-Macaulay ring by Theorem 5.2 , so is $R$ by [21, Proposition 6.1]), so is $\mathrm{H}_{M}^{i}(I R)$. Consequently, we get $\left[\mathrm{H}_{M}^{i}(I R)\right]_{n}=(0)$ for all $n \leq 2-i$ and $n<0$.

We now look at the exact sequence

$$
\left[\mathrm{H}_{M}^{d-1}(I R)\right]_{1-d} \rightarrow\left[\mathrm{H}_{M}^{d-1}(S)\right]_{1-d} \rightarrow\left[\mathrm{H}_{M}^{d}(I T)\right]_{1-d}
$$

of local cohomology modules induced by the canonical exact sequence

$$
0 \rightarrow I T \rightarrow I R \rightarrow S \rightarrow 0
$$

of graded $T$-modules. Then, since $\left[\mathrm{H}_{M}^{d}(I T)\right]_{1-d}=(0)$ by Lemma 2.6 and $\left[\mathrm{H}_{M}^{d-1}(I R)\right]_{1-d}=(0)$ by Claim 1 , we have $\left[\mathrm{H}_{M}^{d-1}(S)\right]_{1-d}=(0)$, whence $\left[\mathrm{H}_{M}^{d-1}(G)\right]_{2-d}=(0)$. Thus, we get $\mathrm{H}_{M}^{d-1}(G)=\left[\mathrm{H}_{M}^{d-1}(G)\right]_{3-d}$.

In the rest of our proof of Proposition 5.3, we have to show that $\left[\mathrm{H}_{M}^{i}(G)\right]_{2-i} \cong \mathrm{H}_{\mathfrak{m}}^{i}(A)$ for $1 \leq i \leq d-1$. Because conditions $\left(\mathrm{C}_{1}\right)$ and $\left(\mathrm{C}_{3}\right)$ are satisfied, we have the canonical exact sequence

$$
\left(\natural^{\prime}\right) \quad 0 \rightarrow A \stackrel{a_{1}}{\rightarrow} A \rightarrow \bar{A} \rightarrow 0
$$

and $a_{1} \mathrm{H}_{\mathfrak{m}}^{1}(A)=(0)$ by [21, Theorem 2.5]. Then applying the local cohomology functor $\mathrm{H}_{\mathfrak{m}}^{i}(*)$ to the exact sequence $\left(\mathfrak{\natural}^{\prime}\right)$, we have $\mathrm{H}_{\mathfrak{m}}^{0}(\bar{A}) \cong \mathrm{H}_{\mathfrak{m}}^{1}(A)$ (see [10, Lemma 2.6], [21, Proof of Lemma 1.7]). Therefore, we get

$$
\mathrm{H}_{M}^{1}(G)=\left[\mathrm{H}_{M}^{1}(G)\right]_{1} \cong\left[\mathrm{H}_{M}^{0}(\mathrm{G}(\bar{I}))\right]_{2} \cong \mathrm{H}_{\mathfrak{m}}^{0}(\bar{A}) \cong \mathrm{H}_{\mathfrak{m}}^{1}(A)
$$

by the exact sequence $(\downarrow)$.

Suppose that $2 \leq i \leq d-1$. Applying local cohomology functors $\mathrm{H}_{M}^{i}(*)$ to exact sequences $\left(\dagger_{1}\right)$ and $\left(\dagger_{2}\right)$, we get monomorphisms

$$
\left[\mathrm{H}_{M}^{i}(G)\right]_{2-i} \hookrightarrow\left[\mathrm{H}_{M}^{i}(R / I R+T)\right]_{2-i} \hookrightarrow\left[\mathrm{H}_{M}^{i}(S)\right]_{1-i} .
$$

Now take the exact sequence

$$
\left[\mathrm{H}_{M}^{i}(I R)\right]_{1-i} \rightarrow\left[\mathrm{H}_{M}^{i}(S)\right]_{1-i} \rightarrow\left[\mathrm{H}_{M}^{i+1}(I T)\right]_{1-i}
$$

induced by the exact sequence

$$
0 \rightarrow I T \rightarrow I R \rightarrow S \rightarrow 0
$$


Then, because $\left[\mathrm{H}_{M}^{i}(I R)\right]_{1-i}=(0)$ by Claim 1 and $\left[\mathrm{H}_{M}^{i+1}(I T)\right]_{1-i} \cong \mathrm{H}_{\mathfrak{m}}^{i}(A)$ by Lemma 2.6, we get

$$
\mathrm{H}_{M}^{i}(G)=\left[\mathrm{H}_{M}^{i}(G)\right]_{2-i} \hookrightarrow \mathrm{H}_{\mathfrak{m}}^{i}(A)
$$

for $2 \leq i \leq d-1$. Then because

$$
\mathbb{I}(A) \leq \mathbb{I}(G)=\sum_{i=1}^{d-1}\left(\begin{array}{c}
d-1 \\
i
\end{array}\right) \ell_{G}\left(\mathrm{H}_{M}^{i}(G)\right) \leq \sum_{i=1}^{d-1}\left(\begin{array}{c}
d-1 \\
i
\end{array}\right) \ell_{A}\left(\mathrm{H}_{\mathfrak{m}}^{i}(A)\right)=\mathbb{I}(A)
$$

(see [5, Proposition 3.2], [21, Corollary 5.2]), we get $\ell_{G}\left(\mathrm{H}_{M}^{i}(G)\right)=\ell_{A}\left(\mathrm{H}_{\mathfrak{m}}^{i}(A)\right)$ for $1 \leq i \leq d-1$. Thus, $\mathrm{H}_{M}^{i}(G)=\left[\mathrm{H}_{M}^{i}(G)\right]_{2-i} \cong \mathrm{H}_{\mathfrak{m}}^{i}(A)$ for all $1 \leq i \leq d-1$. This completes the proof of Proposition 5.3.

Thanks to Proposition 5.3, we can explore the structure of the Sally module $S$ of the ideals $I$ with respect to $Q$ satisfying $2 \mathrm{e}_{0}(I)-\mathrm{e}_{1}(I)+\mathrm{e}_{1}(Q)=$ $2 \ell_{A}(A / I)+\ell_{A}\left(I / I^{2}+Q\right)$ as follows.

Corollary 5.4. Suppose that $S \neq(0)$ and that conditions $\left(\mathrm{C}_{1}\right),\left(\mathrm{C}_{2}\right)$, and $\left(\mathrm{C}_{3}\right)$ are satisfied. Assume that $2 \mathrm{e}_{0}(I)-\mathrm{e}_{1}(I)+\mathrm{e}_{1}(Q)=2 \ell_{A}(A / I)+$ $\ell_{A}\left(I / I^{2}+Q\right)$. Then we have

(1) $\mathrm{e}_{0}(S)=\mathrm{e}_{0}(I)-\ell_{A}(A / I)-\ell_{A}\left(I / I^{2}+Q\right)$,

(2) $\mathrm{e}_{1}(S)=\mathrm{e}_{1}(I)-\mathrm{e}_{0}(I)+\ell_{A}(A / I)$,

(3) $\mathrm{e}_{i}(S)=\mathrm{e}_{i-1}(Q)+\mathrm{e}_{i}(Q)$ for $2 \leq i \leq d-1$,

(4) $\mathrm{H}_{M}^{i}(S)=\left[\mathrm{H}_{M}^{i}(S)\right]_{1-i} \cong \mathrm{H}_{\mathfrak{m}}^{i}(A)$ for $1 \leq i \leq d-1$, and

(5) the a-invariant $\mathrm{a}(S)$ of $S$ is at most $1-d$.

Proof. Recall that $\operatorname{dim}_{T} S=d$ by Lemma 2.3 .

(1), (2), and (3) By Lemma 3.3 and Proposition 3.5, we have

$$
\begin{aligned}
\ell_{A}\left(S_{n-1}\right)= & \ell_{A}\left(I^{n} / I^{n+1}\right)-\left\{\ell_{A}(A / I)+\ell_{A}\left(I / I^{2}+Q\right)\right\}\left(\begin{array}{c}
n+d-1 \\
d-1
\end{array}\right) \\
& +\ell_{A}\left(I / I^{2}+Q\right)\left(\begin{array}{c}
n+d-2 \\
d-2
\end{array}\right)
\end{aligned}
$$

for all $n \geq 0$. On the other hand, we have 


$$
\begin{aligned}
\ell_{A}\left(S_{n-1}\right) \\
=\sum_{i=0}^{d-1}(-1)^{i} \mathrm{e}_{i}(S)\left(\begin{array}{c}
n-1+d-1-i \\
d-1-i
\end{array}\right) \\
=\sum_{i=0}^{d-2}(-1)^{i} \mathrm{e}_{i}(S)\left(\begin{array}{c}
n-1+d-1-i \\
d-1-i
\end{array}\right)+(-1)^{d-1} \mathrm{e}_{d-1}(S) \\
=\sum_{i=0}^{d-2}(-1)^{i} \mathrm{e}_{i}(S)\left\{\left(\begin{array}{c}
n+d-1-i \\
d-1-i
\end{array}\right)-\left(\begin{array}{c}
n+d-2-i \\
d-2-i
\end{array}\right)\right\}+(-1)^{d-1} \mathrm{e}_{d-1}(S) \\
=\sum_{i=0}^{d-1}(-1)^{i} \mathrm{e}_{i}(S)\left(\begin{array}{c}
n+d-1-i \\
d-1-i
\end{array}\right)-\sum_{i=1}^{d-1}(-1)^{i-1} \mathrm{e}_{i-1}(S)\left(\begin{array}{c}
n+d-1-i \\
d-1-i
\end{array}\right) \\
=\mathrm{e}_{0}(S)\left(\begin{array}{c}
n+d-1 \\
d-1
\end{array}\right)+\sum_{i=1}^{d-1}(-1)^{i}\left\{\mathrm{e}_{i}(S)+\mathrm{e}_{i-1}(S)\right\}\left(\begin{array}{c}
n+d-1-i \\
d-1-i
\end{array}\right)
\end{aligned}
$$

for all $n \gg 0$ so that

$$
\begin{aligned}
\mathrm{e}_{0}(S) & =\mathrm{e}_{0}(I)-\left\{\ell_{A}(A / I)+\ell_{A}\left(I / I^{2}+Q\right)\right\}, \\
\mathrm{e}_{1}(S)+\mathrm{e}_{0}(S) & =\mathrm{e}_{1}(I)-\ell_{A}\left(I / I^{2}+Q\right), \\
\mathrm{e}_{i}(S)+\mathrm{e}_{i-1}(S) & =\mathrm{e}_{i}(I) \quad \text { for } 2 \leq i \leq d-1 .
\end{aligned}
$$

Then we have

$$
\begin{aligned}
\mathrm{e}_{1}(S) & =\mathrm{e}_{1}(I)-\ell_{A}\left(I / I^{2}+Q\right)-\mathrm{e}_{0}(S) \\
& =\mathrm{e}_{1}(I)-\ell_{A}\left(I / I^{2}+Q\right)-\left\{\mathrm{e}_{0}(I)-\ell_{A}(A / I)-\ell_{A}\left(I / I^{2}+Q\right)\right\} \\
& =\mathrm{e}_{1}(I)-\mathrm{e}_{0}(I)+\ell_{A}(A / I)
\end{aligned}
$$

and, because $\mathrm{e}_{i}(I)=\mathrm{e}_{i-1}(Q)+\mathrm{e}_{i}(Q)+\mathrm{e}_{i-1}(S)$ for all $2 \leq i \leq d$ by Corollary 2.5 , we have

$$
\begin{aligned}
\mathrm{e}_{i}(S) & =\mathrm{e}_{i}(I)-\mathrm{e}_{i-1}(S) \\
& =\left\{\mathrm{e}_{i-1}(Q)+\mathrm{e}_{i}(Q)+\mathrm{e}_{i-1}(S)\right\}-\mathrm{e}_{i-1}(S)=\mathrm{e}_{i-1}(Q)+\mathrm{e}_{i}(Q)
\end{aligned}
$$

for all $2 \leq i \leq d-1$. 
(4) Thanks to Proposition 3.5, we have exact sequences

$$
\begin{array}{ll}
\left(\dagger_{1}\right) \quad 0 \rightarrow\left(\left(I / I^{2}+Q\right) \otimes F\right)(-1) \rightarrow R / I R+T \rightarrow S(-1) \rightarrow 0, \\
\left(\dagger_{2}\right) \quad 0 \rightarrow F \rightarrow G \rightarrow R / I R+T \rightarrow 0
\end{array}
$$

of graded $T$-modules. Then because $F \cong(A / I)\left[X_{1}, X_{2}, \ldots, X_{d}\right]$ is a polynomial ring over $A / I$ by Lemma 2.2 so that $\left(I / I^{2}+Q\right) \otimes F$ is a maximal Cohen-Macaulay $F$-module, we obtain

$$
\mathrm{H}_{M}^{i}(G) \cong \mathrm{H}_{M}^{i}(R / I R+T) \cong \mathrm{H}_{M}^{i}(S)(-1)
$$

as graded $T$-modules for $0 \leq i \leq d-2$. Therefore, since $\mathrm{H}_{M}^{i}(G)=$ $\left[\mathrm{H}_{M}^{i}(G)\right]_{2-i} \cong \mathrm{H}_{\mathfrak{m}}^{i}(A)$ by Proposition $5.3(2)$, we get $\mathrm{H}_{M}^{i}(S)=\left[\mathrm{H}_{M}^{i}(S)\right]_{1-i} \cong$ $\mathrm{H}_{\mathfrak{m}}^{i}(A)$ for $1 \leq i \leq d-2$.

Let us look at the exact sequences

$$
\begin{aligned}
& \left(\dagger_{1}\right)^{\prime} \quad 0 \rightarrow \mathrm{H}_{M}^{d-1}(R / I R+T) \rightarrow \mathrm{H}_{M}^{d-1}(S)(-1) \rightarrow \mathrm{H}_{M}^{d}\left(\left(I / I^{2}+Q\right) \otimes F\right)(-1), \\
& \left(\dagger_{2}\right)^{\prime} \quad 0 \rightarrow \mathrm{H}_{M}^{d-1}(G) \rightarrow \mathrm{H}_{M}^{d-1}(R / I R+T) \rightarrow \mathrm{H}_{M}^{d}(F)
\end{aligned}
$$

of local cohomology modules induced by the exact sequences $\left(\dagger_{1}\right)$ and $\left(\dagger_{2}\right)$. Then because $\mathrm{H}_{M}^{d-1}(G)=\left[\mathrm{H}_{M}^{d-1}(G)\right]_{3-d}$ by Proposition 5.3(2) and $\left[\mathrm{H}_{M}^{d}(F)\right]_{n}=(0)$ for all $n>-d$, we get $\left[\mathrm{H}_{M}^{d-1}(R / I R+T)\right]_{n}=(0)$ for all $n \geq$ $4-d$ by the exact sequence $\left(\dagger_{2}\right)^{\prime}$. Then since $\left[\mathrm{H}_{M}^{d}\left(\left(I / I^{2}+Q\right) \otimes F\right)(-1)\right]_{n}=$ (0) for all $n \geq 2-d$ and by the exact sequence $\left(\dagger_{1}\right)^{\prime}$, we obtain $\left[\mathrm{H}_{M}^{d-1}(S)\right]_{n}=$ (0) for all $n \geq 3-d$.

From the exact sequence

$$
\mathrm{H}_{M}^{d-1}(I R) \rightarrow \mathrm{H}_{M}^{d-1}(S) \rightarrow \mathrm{H}_{M}^{d}(I T)
$$

of local cohomology modules induced by the exact sequence

$$
0 \rightarrow I T \rightarrow I R \rightarrow S \rightarrow 0
$$

of graded $T$-modules, it follows that $\mathrm{H}_{M}^{d-1}(S)=\left[\mathrm{H}_{M}^{d-1}(S)\right]_{2-d}$ because $\left[\mathrm{H}_{M}^{d}(I T)\right]_{n}=(0)$ for all $n \leq 1-d$ by Lemma 2.6 and $\left[\mathrm{H}_{M}^{d-1}(I R)\right]_{n}=(0)$ for all $n \leq 1-d<0$ by Claim 1 in our proof of Theorem 5.3. Therefore, we get isomorphisms

$$
\mathrm{H}_{M}^{d-1}(S) \cong\left[\mathrm{H}_{M}^{d-1}(S)\right]_{2-d} \cong\left[\mathrm{H}_{M}^{d-1}(G)\right]_{3-d} \cong \mathrm{H}_{\mathfrak{m}}^{d-1}(A)
$$

by the exact sequences $\left(\dagger_{1}\right)^{\prime},\left(\dagger_{2}\right)^{\prime}$, and Proposition 5.3(2).

(5) Since $\mathrm{a}(G) \leq 2-d$ by Proposition $5.3(3)$, we get $\mathrm{a}(S) \leq 1-d$ by the exact sequences $\left(\dagger_{1}\right)$ and $\left(\dagger_{2}\right)$.

We are now in a position to prove the last assertions in Theorem 1.1. 
Proof of the last assertions in Theorem 1.1. Assertion (i). Thanks to Proposition 5.3(1), we may assume that $A$ is a Buchsbaum ring. Then since $I^{3} \subseteq Q I^{2}+W$ by Theorem 4.3 and $\mathfrak{m} \cdot W=(0), I^{4}=Q I^{3}$ holds true. Therefore, $I^{n} \cap W \subseteq Q \cap W=(0)$ for all $n \geq 4$ by [21, Corollary 2.3]. Thus, we get the required assertions by Proposition 5.3(1).

Assertions (ii), (iii). See Proposition 5.3(2),(3).

Assertions (iv), ( $v$ ). When $I^{2} \subseteq Q$, then $A$ is a Cohen-Macaulay local ring and $I^{2}=Q I$ holds true by Remark 5.1. Then we have $\mathrm{e}_{1}(I)=\mathrm{e}_{0}(I)-$ $\ell_{A}(A / I)$ and $\mathrm{e}_{i}(I)=0$ for $2 \leq i \leq d($ see $[14$, Theorem 2.1], [7, Corollary 2.3]), and $\mathrm{e}_{i}(Q)=0$ for all $1 \leq i \leq d$, whence our assertions hold true. Thus, we may assume that $I^{2} \subseteq / Q$, whence $S \neq(0)$.

Put $C=A / W$. Then all conditions $\left(\mathrm{C}_{1}\right),\left(\mathrm{C}_{2}\right)$, and $\left(\mathrm{C}_{3}\right)$ are satisfied for the ring $C$ and the ideals $I C$ and $Q C$, and we have $\mathrm{e}_{i}(I)=\mathrm{e}_{i}(I C)$, $\mathrm{e}_{i}(Q)=\mathrm{e}_{i}(Q C)$ for all $0 \leq i \leq d-1, \mathrm{e}_{d}(I)=\mathrm{e}_{d}(I C)+(-1)^{d} \ell_{A}(W), \mathrm{e}_{d}(Q)=$ $\mathrm{e}_{d}(Q C)+(-1)^{d} \ell_{A}(W)$, and $\ell_{A}(A / I)=\ell_{A}(C / I C)$. We also have $2 \mathrm{e}_{0}(I C)-$ $\mathrm{e}_{1}(I C)+\mathrm{e}_{1}(Q C)=2 \ell_{A}(C / I C)+\ell_{A}\left(I C / I^{2} C+Q C\right)$ by Lemma 4.2 , whence passing to the ring $C$, we may assume that condition $\left(\mathrm{C}_{3}\right)$ is satisfied.

Thanks to Corollary 5.4, we have

$$
\mathrm{e}_{1}(S)=\mathrm{e}_{1}(I)-\mathrm{e}_{0}(I)+\ell_{A}(A / I) \quad \text { and } \quad \mathrm{e}_{i}(S)=\mathrm{e}_{i-1}(Q)+\mathrm{e}_{i}(Q)
$$

for all $2 \leq i \leq d-1$. Therefore, by Corollary 2.5, we have

$$
\begin{aligned}
& \mathrm{e}_{2}(I)=\mathrm{e}_{1}(Q)+\mathrm{e}_{2}(Q)+\mathrm{e}_{1}(S)=\mathrm{e}_{1}(Q)+\mathrm{e}_{2}(Q)+\mathrm{e}_{1}(I)-\mathrm{e}_{0}(I)+\ell_{A}(A / I), \\
& \mathrm{e}_{i}(I)=\mathrm{e}_{i-1}(Q)+\mathrm{e}_{i}(Q)+\mathrm{e}_{i-1}(S)=\mathrm{e}_{i-2}(Q)+2 \mathrm{e}_{i-1}(Q)+\mathrm{e}_{i}(Q)
\end{aligned}
$$

for all $3 \leq i \leq d$.

Assertion (vi). We proceed by induction on $d$. Suppose that $d=1$. Then since $\mathfrak{m} \cdot W$, we have $M \cdot \mathrm{H}_{M}^{0}(G)=(0)$ by assertion (i). Thus, $G$ is a Buchsbaum ring (see [20, I. Proposition 2.12]).

Assume that $d \geq 2$ and that our assertion holds true for $d-1$. Put $C=A / W$. Then all conditions $\left(\mathrm{C}_{1}\right),\left(\mathrm{C}_{2}\right)$, and $\left(\mathrm{C}_{3}\right)$ are satisfied for the $\operatorname{ring} C$ and the ideals $I C$ and $Q C$, and we have $2 \mathrm{e}_{0}(I C)-\mathrm{e}_{1}(I C)+\mathrm{e}_{1}(Q C)=$ $2 \ell_{A}(C / I C)+\ell_{A}\left(I C / I^{2} C+Q C\right)$ by Lemma 4.2. Then we have $\operatorname{depth} \mathrm{G}(I C)>$ 0 and

$$
\mathrm{H}_{M}^{i}(\mathrm{G}(I C))=\left[\mathrm{H}_{M}^{i}(\mathrm{G}(I C))\right]_{2-i} \cong \mathrm{H}_{\mathfrak{m}}^{i}(C)
$$

for $1 \leq i \leq d-1$ by assertion (ii).

Let $D=C / a_{1} C$, and put $f=a_{1} t$. Then $f$ is a $\mathrm{G}(I C)$-regular element, and all conditions $\left(\mathrm{C}_{1}\right)$ and $\left(\mathrm{C}_{2}\right)$ are satisfied for the ring $D$ and the ideals 
$I D$ and $Q D$. We also have $2 \mathrm{e}_{0}(I D)-\mathrm{e}_{1}(I D)+\mathrm{e}_{1}(Q D)=2 \ell_{A}(D / I D)+$ $\ell_{A}\left(I D / I^{2} D+Q D\right)$ by Lemma 4.1 .

Then the hypothesis of induction on $d$ says that $\mathrm{G}(I D)$ is a Buchsbaum ring. Because $f=a_{1} t$ is a $\mathrm{G}(I C)$-regular element, $\mathrm{G}(I D) \cong \mathrm{G}(I C) / f \mathrm{G}(I C)$, and $f \mathrm{H}_{M}^{i}(\mathrm{G}(I C))=(0)$ for $1 \leq i \leq d-1, \mathrm{G}(I C)$ is also a Buchsbaum ring by [20, I. Proposition 2.19]. Furthermore, the kernel of the graded homomorphism $G \rightarrow \mathrm{G}(I C)$ coincides with $\mathrm{H}_{M}^{0}(G)$. Thus, we get that $G / \mathrm{H}_{M}^{0}(G)$ is a Buchsbaum ring.

Let $V=\mathfrak{m}+I t \subseteq R$. Then, because the residue class field $A / \mathfrak{m}$ is infinite, we may choose a system of generators $\xi_{1}, \xi_{2}, \ldots, \xi_{\ell}$ of $V$ such that $\left\{\xi_{i}\right\}_{i \in \Lambda}$ form a system of parameters for $G_{M}$ for any subset $\Lambda \subseteq\{1,2, \ldots, \ell\}$ with $d$ elements. In order to prove the Buchsbaumness of $G$, it is enough to show that

$$
\left(\left\{\xi_{i}\right\}_{i \in \Lambda}\right) G \cap \mathrm{H}_{M}^{0}(G)=(0)
$$

for any $\Lambda$ stated above (see [20, I. Proposition 2.22]).

We may take $\Lambda=\{1,2, \ldots, d\}$ for simplicity, and let $\xi_{i}=x_{i}-b_{i} t$ with $x_{i} \in$ $\mathfrak{m}$ and $b_{i} \in I$ for $1 \leq i \leq d$. Put $\mathfrak{q}=\left(b_{1}, b_{2}, \ldots, b_{d}\right)$. Since $\left(\xi_{1}, \xi_{2}, \ldots, \xi_{d}\right) G+$ $\mathfrak{m} G=\left(b_{1} t, b_{2} t, \ldots, b_{d} t\right) G+\mathfrak{m} G, b_{1} t, b_{2} t, \ldots, b_{d} t$ forms a system of parameters for $G / \mathfrak{m} G$. Hence, $\mathfrak{q}=\left(b_{1}, b_{2}, \ldots, b_{d}\right)$ is a reduction of $I$. Then we have that condition $\left(\mathrm{C}_{1}\right)$ is satisfied for the ideal $\mathfrak{q}=\left(b_{1}, b_{2}, \ldots, b_{d}\right)$ by our assumption that $A$ is a Buchsbaum local ring. Since the Castelnuovo-Mumford regularity

$$
\operatorname{reg} \mathrm{G}(I C):=\max \left\{n+i \mid\left[\mathrm{H}_{M}^{i}(\mathrm{G}(I C))\right]_{n} \neq(0), i, n \in \mathbb{Z}\right\}
$$

of $\mathrm{G}(I C)$ is at most 2 by assertions (i) and (ii), we get $I^{3} C=\mathfrak{q} I^{2} C$, whence $I^{3} \subseteq \mathfrak{q} I^{2}+W$ (see [22, Proposition 3.2])

Let $g_{i}=b_{i} t$ for $1 \leq i \leq d$. We then have the following claim.

Claim 2. We have that $g_{1}, g_{2}, \ldots, g_{d}$ is standard on $G$.

Proof. Since $G$ is a generalized Cohen-Macaulay ring with $\mathbb{I}(G)=\mathbb{I}(A)$ by assertions (i) and (ii), we have

$$
\begin{aligned}
\mathbb{I}(G) & \geq \ell_{G}\left(G /\left(g_{1}, g_{2}, \ldots, g_{d}\right) G\right)-\mathrm{e}_{0}\left(g_{1}, g_{2}, \ldots, g_{d} ; G\right) \\
& \geq \ell_{A}(A / \mathfrak{q})-\mathrm{e}_{0}(\mathfrak{q})=\mathbb{I}(A)=\mathbb{I}(G),
\end{aligned}
$$

where $\mathrm{e}_{0}\left(g_{1}, g_{2}, \ldots, g_{d} ; G\right)$ denotes the multiplicity of $G$ with respect to $\left(g_{1}, g_{2}, \ldots, g_{d}\right) G$. Therefore, $\mathbb{I}(G)=\ell_{G}\left(G /\left(g_{1}, g_{2}, \ldots, g_{d}\right) G\right)-\mathrm{e}_{0}\left(g_{1}, g_{2}, \ldots\right.$, $\left.g_{d} ; G\right)$, whence $g_{1}, g_{2}, \ldots, g_{d}$ is standard on $G$ (see [21, Theorem 2.1]). 
Take any $\varphi \in\left(\xi_{1}, \xi_{2}, \ldots, \xi_{d}\right) G \cap \mathrm{H}_{M}^{0}(G)$. Then we can write $\varphi=\overline{w_{2} t^{2}+w_{3} t^{3}}$ with $w_{2} \in W$ and $w_{3} \in W \cap I^{3}$ by assertion (i), where $\overline{w_{2} t^{2}+w_{3} t^{3}}$ denotes the image of $w_{2} t^{2}+w_{3} t^{3} \in R$ in $G$.

Let us write $\varphi=\sum_{i=1}^{d} \xi_{i} \overline{\eta_{i}}$ with $\eta_{i} \in R$ for $1 \leq i \leq d$, where $\overline{\eta_{i}}$ denotes the image of $\eta_{i}$ in $G$. Taking $N \gg 0$, we write $\eta_{i}=\sum_{j=0}^{N} \eta_{i_{j}} t^{j}$ with $\eta_{i_{j}} \in I^{j}$ for $0 \leq j \leq N$ and $1 \leq i \leq d$. Then we may express $\eta_{i_{j}} \in \mathfrak{q} I^{j-1}$ for $3 \leq j \leq N$ and $1 \leq i \leq d$, because $\xi_{i} \in \mathfrak{m} A[t], I^{3} \subseteq \mathfrak{q} I^{2}+W$, and $\mathfrak{m} \cdot W=(0)$. Then since we have

$$
\begin{aligned}
\overline{w_{2} t^{2}+w_{3} t^{3}} & =\sum_{i=1}^{d} \xi_{i} \overline{\eta_{i}} \\
& =\sum_{i=1}^{d} \xi_{i}\left(\sum_{j=0}^{N} \overline{\eta_{i_{j}} t^{j}}\right) \\
& =\sum_{j=0}^{N} \sum_{i=1}^{d}\left(x_{i}-b_{i} t\right) \overline{\eta_{i_{j}} t^{j}} \\
& =\sum_{j=0}^{N} \sum_{i=1}^{d} \overline{x_{i} \eta_{i_{j}} t^{j}}-\sum_{j=0}^{N} \sum_{i=1}^{d} \overline{b_{i} \eta_{i_{j}} t^{j+1}} \\
& =\sum_{i=1}^{d} \overline{x_{i} \eta_{i_{0}}}+\sum_{j=1}^{N} \sum_{i=1}^{d} \overline{\left(x_{i} \eta_{i_{j}}-b_{i} \eta_{i_{j-1}}\right) t^{j}}-\sum_{i=1}^{d} \overline{b_{i} \eta_{i_{N}} t^{N+1}}
\end{aligned}
$$

in $G$, we get the following equations:

$$
\begin{aligned}
& w_{2}-\sum_{i=1}^{d}\left(x_{i} \eta_{i_{2}}-b_{i} \eta_{i_{1}}\right) \in I^{3}, \\
& w_{3}-\sum_{i=1}^{d}\left(x_{i} \eta_{i_{3}}-b_{i} \eta_{i_{2}}\right) \in I^{4}, \\
& \sum_{i=1}^{d}\left(x_{i} \eta_{i_{j}}-b_{i} \eta_{i_{j-1}}\right) \in I^{j+1} \quad \text { for } 4 \leq j \leq N,
\end{aligned}
$$

and

$$
\sum_{i=1}^{d} b_{i} \eta_{i_{N}} \in I^{N+2}
$$


Let us now look at the second equality,

$$
w_{3}-\sum_{i=1}^{d}\left(x_{i} \eta_{i_{3}}-b_{i} \eta_{i_{2}}\right) \in I^{4}=\mathfrak{q} I^{3}
$$

Then because $x_{i} \eta_{i_{3}} \in \mathfrak{m} I^{3} \subseteq \mathfrak{m}\left(\mathfrak{q} I^{2}+W\right) \subseteq \mathfrak{q}$ and condition $\left(\mathrm{C}_{1}\right)$ is satisfied, $w_{3} \in \mathfrak{q} \cap W=(0)$ by [21, Corollary 2.3], whence $w_{3}=0$.

It is now enough to show that $w_{2} \in I^{3}$. We need the following claim.

Claim 3. There exist elements $y_{\alpha_{\beta}}^{(j)} \in I^{j}$ for $2 \leq j \leq N$ and $1 \leq \alpha<\beta \leq d$ such that

$$
\sum_{i=1}^{d} b_{i}\left(\eta_{i_{j}}+\sum_{\alpha<i} x_{\alpha} y_{\alpha_{i}}^{(j)}-\sum_{i<\beta} x_{\beta} y_{i_{\beta}}^{(j)}\right) \in I^{j+2} .
$$

Proof of Claim 3. We proceed by descending induction on $j$. When $j=$ $N$, we may take $y_{\alpha_{\beta}}^{(j)}=0$ for all $1 \leq \alpha<\beta \leq d$.

Assume that $j \leq N-1$ and that our assertion holds true for $j+1$. Then by the hypothesis of induction $j$ there exist elements $y_{\alpha_{\beta}}^{(j+1)} \in I^{j+1}$ for all $1 \leq \alpha<\beta \leq d$ such that

$$
\sum_{i=1}^{d} b_{i}\left(\eta_{i_{j+1}}+\sum_{\alpha<i} x_{\alpha} y_{\alpha_{i}}^{(j+1)}-\sum_{i<\beta} x_{\beta} y_{i_{\beta}}^{(j+1)}\right) \in I^{j+3} .
$$

Then since $x_{i} \in \mathfrak{m}$ and $x_{i} y_{\alpha_{\beta}}^{(j+1)} \in x_{i} I^{j+1} \subseteq x_{i}\left(\mathfrak{q} I^{j}+W\right)=x_{i} \mathfrak{q} I^{j}$, we may take $y_{\alpha_{\beta}}^{(j+1)} \in \mathfrak{q} I^{j}$ for all $1 \leq \alpha<\beta \leq d$. Put

$$
v_{i}^{(j+1)}=\eta_{i_{j+1}}+\sum_{\alpha<i} x_{\alpha} y_{\alpha_{i}}^{(j+1)}-\sum_{i<\beta} x_{\beta} y_{i_{\beta}}^{(j+1)}
$$

for $1 \leq i \leq d$. Let $K_{\bullet}=\mathrm{K}_{\bullet}\left(g_{1}, g_{2}, \ldots, g_{d} ; G\right)$ be the Koszul complex with the differential maps $\partial_{p}: K_{p} \rightarrow K_{p-1}$, and let $T_{1}, T_{2}, \ldots, T_{d}$ be the free basis of $K_{1}$. Put $\sigma=\sum_{i=1}^{d} \overline{v_{i}^{(j+1)} t^{j+1}} T_{i}$. We then have

$$
\partial_{1}(\sigma)=\sum_{i=1}^{d} \overline{g_{i} v_{i}^{(j+1)} t^{j+1}}=\overline{\left(\sum_{i=1}^{d} b_{i} v_{i}^{(j+1)}\right) t^{j+2}}=0
$$


in $G$ because $\sum_{i=1}^{d} b_{i} v_{i}^{(j+1)} \in I^{j+3}$. On the other hand, since $v_{i}^{(j+1)} \in \mathfrak{q} I^{j}$ we have $\sigma \in\left(g_{1}, g_{2}, \ldots, g_{d}\right) K_{1}$. Hence, $\sigma \in\left(g_{1}, g_{2}, \ldots, g_{d}\right) K_{1} \cap Z_{1}(K)=B_{1}(K)$ because $g_{1}, g_{2}, \ldots, g_{d}$ forms a $d$-sequence on $G$ by Claim 2 , where $Z_{1}(K):=$ $\operatorname{Ker} \partial_{1}$ and $B_{1}(Z):=\operatorname{Im} \partial_{2}$. Therefore, there exist elements $y_{\alpha_{\beta}}^{(j)} \in I^{j}$ for $1 \leq \alpha<\beta \leq d$ such that

$$
\partial_{2}\left(\sum_{\alpha<\beta} \overline{y_{\alpha \beta}^{(j)} t^{j}} T_{\alpha} \wedge T_{\beta}\right)=\sigma .
$$

Then we have

$$
\begin{aligned}
\sigma & =\partial_{2}\left(\sum_{\alpha<\beta} \overline{y_{\alpha_{\beta}}^{(j)} t^{j}} T_{\alpha} \wedge T_{\beta}\right) \\
& =\sum_{\alpha<\beta} \overline{g_{\alpha} y_{\alpha_{\beta}}^{(j)} t^{j}} T_{\beta}-\sum_{\alpha<\beta} \overline{g_{\beta} y_{\alpha_{\beta}}^{(j)} t^{j}} T_{\alpha} \\
& =\sum_{\alpha<\beta} \overline{b_{\alpha} y_{\alpha_{\beta}}^{(j)} t^{j+1}} T_{\beta}-\sum_{\alpha<\beta} \overline{b_{\beta} y_{\alpha_{\beta}}^{(j)} t^{j+1}} T_{\alpha} \\
& =\sum_{i=1}^{d} \overline{\left(\sum_{\alpha<i} b_{\alpha} y_{\alpha_{i}}^{(j)}-\sum_{i<\beta} b_{\beta} y_{i_{\beta}}^{(j)}\right) t^{j+1}} T_{i} .
\end{aligned}
$$

Hence, we have

$$
\overline{v_{i}^{(j+1)} t^{j+1}}=\overline{\left(\sum_{\alpha<i} b_{\alpha} y_{\alpha_{i}}^{(j)}-\sum_{i<\beta} b_{\beta} y_{i_{\beta}}^{(j)}\right) t^{j+1}}
$$

in $G$ so that

$$
v_{i}^{(j+1)}-\left(\sum_{\alpha<i} b_{\alpha} y_{\alpha_{i}}^{(j)}-\sum_{i<\beta} b_{\beta} y_{i_{\beta}}^{(j)}\right) \in I^{j+2}
$$

for all $1 \leq i \leq d$. Then since $\sum_{i=1}^{d} x_{i} v_{i}^{(j+1)}=\sum_{i=1}^{d} x_{i} \eta_{i_{j+1}}$, we have the following:

$$
\begin{aligned}
& \sum_{i=1}^{d} x_{i}\left(v_{i}^{(j+1)}-\sum_{\alpha<i} b_{\alpha} y_{\alpha_{i}}^{(j)}-\sum_{i<\beta} b_{\beta} y_{i_{\beta}}^{(j)}\right) \in I^{j+2} \\
& \quad=\sum_{i=1}^{d} x_{i} v_{i}^{(j+1)}-\left(\sum_{i=1}^{d} \sum_{\alpha<i} x_{i} b_{\alpha} y_{\alpha_{i}}^{(j)}-\sum_{i=1}^{d} \sum_{i<\beta} x_{i} b_{\beta} y_{i_{\beta}}^{(j)}\right)
\end{aligned}
$$




$$
\begin{aligned}
& =\sum_{i=1}^{d} x_{i} \eta_{i_{j+1}}-\left(\sum_{i=1}^{d} \sum_{i<\beta} x_{\beta} b_{i} y_{i_{\beta}}^{(j)}-\sum_{i=1}^{d} \sum_{\alpha<i} x_{\alpha} b_{i} y_{\alpha_{i}}^{(j)}\right) \\
& =\sum_{i=1}^{d} x_{i} \eta_{i_{j+1}}-\sum_{i=1}^{d} b_{i}\left(\sum_{i<\beta} x_{\beta} y_{i_{\beta}}^{(j)}-\sum_{\alpha<i} x_{\alpha} y_{\alpha_{i}}^{(j)}\right) .
\end{aligned}
$$

Therefore, because $\sum_{i=1}^{d}\left(x_{i} \eta_{i_{j+1}}-b_{i} \eta_{i_{j}}\right) \in I^{j+2}$, we get

$$
\sum_{i=1}^{d} b_{i}\left(\eta_{i_{j}}+\sum_{\alpha<i} x_{\alpha} y_{\alpha_{i}}^{(j)}-\sum_{i<\beta} x_{\beta} y_{i_{\beta}}^{(j)}\right) \in I^{j+2}
$$

as required.

Thanks to Claim 3, there exist elements $y_{\alpha_{\beta}}^{(2)} \in I^{2}$ for $1 \leq \alpha<\beta \leq d$ such that

$$
\sum_{i=1}^{d} b_{i}\left(\eta_{i_{2}}+\sum_{\alpha<i} x_{\alpha} y_{\alpha_{i}}^{(2)}-\sum_{i<\beta} x_{\beta} y_{i_{\beta}}^{(2)}\right) \in I^{4} .
$$

Put

$$
v_{i}=v_{i}^{(2)}=\eta_{i_{2}}+\sum_{\alpha<i} x_{\alpha} y_{\alpha_{i}}^{(2)}-\sum_{i<\beta} x_{\beta} y_{i_{\beta}}^{(2)}
$$

for $1 \leq i \leq d$. Then we have $\sum_{i=1}^{d} b_{i} v_{i} \in I^{4}=\mathfrak{q} I^{3}$. Therefore, there exist $v_{i}^{\prime} \in$ $I^{3}$ with $1 \leq i \leq d$ such that $\sum_{i=1}^{d} b_{i} v_{i}=\sum_{i=1}^{d} b_{i} v_{i}^{\prime}$, whence we get $\sum_{i=1}^{d} b_{i}\left(v_{i}-\right.$ $\left.v_{i}^{\prime}\right)=0$. Then for each $1 \leq i \leq d$ we have

$$
v_{i}-v_{i}^{\prime} \in\left[\left(b_{1}, b_{2}, \ldots, \check{b}_{i}, \ldots, b_{d}\right): b_{i}\right]=\left[\left(b_{1}, b_{2}, \ldots, \check{b_{i}}, \ldots, b_{d}\right): \mathfrak{m}\right]
$$

so that $x_{i}\left(v_{i}-v_{i}^{\prime}\right) \in \mathfrak{q}$. Since $x_{i} v_{i}^{\prime} \in \mathfrak{m} I^{3} \subseteq \mathfrak{m}\left(\mathfrak{q} I^{2}+W\right) \subseteq \mathfrak{q}$, we get $x_{i} v_{i} \in \mathfrak{q}$.

On the other hand, since $\sum_{i=1}^{d} x_{i} v_{i}=\sum_{i=1}^{d} x_{i} \eta_{i_{2}}$, we have

$$
\sum_{i=1}^{d}\left(x_{i} \eta_{i_{2}}-b_{i} \eta_{i_{1}}\right)=\sum_{i=1}^{d}\left(x_{i} v_{i}-b_{i} \eta_{i_{1}}\right) \in \mathfrak{q} .
$$

Put $q=\sum_{i=1}^{d}\left(x_{i} \eta_{i_{2}}-b_{i} \eta_{i_{1}}\right)$; then because $w_{2}-q \in I^{3} \subseteq \mathfrak{q} I^{2}+W$, we may write $w_{2}-q=q^{\prime}+w^{\prime}$ with $q^{\prime} \in \mathfrak{q} I^{2}$ and $w^{\prime} \in W$. Then as $w_{2}-w^{\prime}=q+q^{\prime} \in$ $\mathfrak{q} \cap W=(0)$ by [21, Corollary 2.3], we get $q=-q^{\prime} \in \mathfrak{q} I^{2}$. Thus, because $w_{2}-q \in I^{3}$, we get $w_{2} \in I^{3}$. This completes the proof of assertion (vi) and that of Theorem 1.1 as well. 


\section{§6. An example}

In this section, we construct one example of an $\mathfrak{m}$-primary ideal $I$ which satisfies $2 \mathrm{e}_{0}(I)-\mathrm{e}_{1}(I)+\mathrm{e}_{1}(Q)=2 \ell_{A}(A / I)+\ell_{A}\left(I / I^{2}+Q\right)$ in a Buchsbaum local ring. Our goal is the following.

TheOREM 6.1. Let $\ell>0$ and $d \geq 2$ be integers. Then there exists an $\mathfrak{m}$-primary ideal $I$ in a Buchsbaum local ring $(A, \mathfrak{m})$ such that

$$
d=\operatorname{dim} A, \quad \mathrm{H}_{\mathfrak{m}}^{i}(A)=(0) \quad \text { for } i \neq 1, d, \quad \ell_{A}\left(\mathrm{H}_{\mathfrak{m}}^{1}(A)\right)=\ell,
$$

and

$$
2 \mathrm{e}_{0}(I)-\mathrm{e}_{1}(I)+\mathrm{e}_{1}(Q)=2 \ell_{A}(A / I)+\ell_{A}\left(I / I^{2}+Q\right)
$$

for some reduction $Q=\left(a_{1}, a_{2}, \ldots, a_{d}\right)$ of $I$.

To construct the necessary example we need some techniques which are due to $[4$, Section 6$]$. Let us begin with the following.

Let $m, \ell>0$, and $d \geq 2$ be integers. Let

$$
U=k\left[\left\{X_{i}\right\}_{1 \leq i \leq m},\left\{Y_{j}\right\}_{1 \leq j \leq \ell},\left\{V_{j_{k}}\right\}_{1 \leq j \leq \ell, 1 \leq k \leq d},\left\{Z_{k}\right\}_{1 \leq k \leq d}\right]
$$

be the polynomial ring with $m+\ell+\ell d+d$ indeterminates over an infinite field $k$, and let

$$
\begin{aligned}
\mathfrak{a}= & \left(\left\{X_{i}\right\}_{1 \leq i \leq m},\left\{Y_{j}\right\}_{1 \leq j \leq \ell},\left\{V_{j_{k}}\right\}_{1 \leq j \leq \ell, 1 \leq k \leq d}\right) \cdot\left(\left\{X_{i}\right\}_{1 \leq i \leq m},\left\{Y_{j}\right\}_{1 \leq j \leq \ell}\right) \\
& +\left(V_{j_{k}} V_{j^{\prime}{ }^{\prime}} \mid 1 \leq j, j^{\prime} \leq \ell, 1 \leq k, k^{\prime} \leq d, j \neq j^{\prime} \text { or } k \neq k^{\prime}\right) \\
& +\left(V_{j_{k}}^{2}-Y_{j} Z_{k} \mid 1 \leq j \leq \ell, 1 \leq k \leq d\right) .
\end{aligned}
$$

We put $C=U / \mathfrak{a}$ and denote the images of $X_{i}, Y_{j}, V_{j_{k}}$, and $Z_{k}$ in $C$ by $x_{i}$, $y_{j}, v_{j_{k}}$, and $a_{k}$, respectively. Then $\operatorname{dim} C=d$, since $\sqrt{\mathfrak{a}}=\left(X_{i}, Y_{j}, V_{j_{k}} \mid 1 \leq\right.$ $i \leq m, 1 \leq j \leq \ell, 1 \leq k \leq d)$. Let $\mathcal{M}=C_{+}:=\left(x_{i}, y_{j}, v_{j_{k}}, a_{k} \mid 1 \leq i \leq m, 1 \leq\right.$ $j \leq \ell, 1 \leq k \leq d)$ be the graded maximal ideal in $C$. Let $\Lambda$ be a subset of $\{1,2, \ldots, m\}$. We put

$$
\mathfrak{q}=\left(a_{i} \mid 1 \leq i \leq d\right)
$$

and

$$
J_{\Lambda}=\mathfrak{q}+\left(x_{\alpha} \mid \alpha \in \Lambda\right)+\left(v_{j_{k}} \mid 1 \leq j \leq \ell, 1 \leq k \leq d\right)
$$


Then $\mathcal{M}^{2}=\mathfrak{q} \mathcal{M}, J_{\Lambda}^{2}=\mathfrak{q} J_{\Lambda}+\mathfrak{q}\left(y_{1}, y_{2}, \ldots, y_{\ell}\right)$, and $J_{\Lambda}^{3}=\mathfrak{q} J_{\Lambda}^{2}$, whence $\mathfrak{q}$ is a reduction of both $\mathcal{M}$ and $J_{\Lambda}$, and $a_{1}, a_{2}, \ldots, a_{d}$ is a homogeneous system of parameters for the graded ring $C$.

Let $B=C_{\mathcal{M}}$, and let $\mathfrak{n}=\mathcal{M} B$ denote the maximal ideal of $B$. We then have the following.

THEOREM 6.2. The following assertions hold true:

(1) $B$ is a Cohen-Macaulay local ring with $\operatorname{dim} B=d$;

(2) $\mathrm{e}_{0}(\mathfrak{q} B)=\mathrm{e}_{0}\left(J_{\Lambda} B\right)=m+\ell d+\ell+1$;

(3) $\mathrm{e}_{1}\left(J_{\Lambda} B\right)=\mathrm{e}_{0}\left(J_{\Lambda} B\right)-\ell_{B}\left(B / J_{\Lambda} B\right)+\ell=\sharp \Lambda+\ell d+\ell$, where $\sharp \Lambda$ denotes the number of elements of the set $\Lambda$;

(4) $\mathrm{e}_{i}\left(J_{\Lambda} B\right)=0$ for all $2 \leq i \leq d$;

(5) $\mathrm{G}\left(J_{\Lambda} B\right)$ is a Buchsbaum ring with depth $\mathrm{G}\left(J_{\Lambda} B\right)=0$ and $\mathbb{I}\left(\mathrm{G}\left(J_{\Lambda} B\right)\right)=$ $\ell d$; and

(6) $\mathrm{R}\left(J_{\Lambda} B\right)$ and $\mathrm{R}^{\prime}\left(J_{\Lambda} B\right)$ are Buchsbaum rings with $\mathbb{I}\left(\mathrm{R}\left(J_{\Lambda} B\right)\right)=$ $\mathbb{I}\left(\mathrm{R}^{\prime}\left(J_{\Lambda} B\right)\right)=\ell d$.

We divide the proof of Theorem 6.2 into a few steps. Let us begin with the following.

Proposition 6.3. Let $\mathfrak{p}=\left(X_{i} \mid 1 \leq i \leq m\right)+\left(Y_{j} \mid 1 \leq j \leq \ell\right)+\left(V_{j_{k}} \mid 1 \leq\right.$ $j \leq \ell, 1 \leq k \leq d)$ in $U$. Then $\ell_{C_{\mathfrak{p}}}\left(C_{\mathfrak{p}}\right)=m+\ell d+\ell+1$.

Proof. Let $\widetilde{U}=U\left[\left\{1 / Z_{k}\right\}_{1 \leq k \leq d}\right]$, and put $\widetilde{k}=k\left[\left\{Z_{k}\right\}_{1 \leq k \leq d},\left\{1 / Z_{k}\right\}_{1 \leq k \leq d}\right]$ in $\widetilde{U}$. Let $X_{i}^{\prime}=X_{i} / Z_{1}(1 \leq i \leq m), Y_{j}^{\prime}=Y_{j} / Z_{1} \quad(1 \leq j \leq \ell)$, and $\bar{V}_{j_{k}}^{\prime}=$ $V_{j_{k}} / Z_{1} \quad(1 \leq j \leq \ell, \quad 1 \leq k \leq d)$. Then $\left\{X_{i}^{\prime}\right\}_{1 \leq i \leq m}, \quad\left\{Y_{j}^{\prime}\right\}_{1 \leq j \leq \ell}$, and $\left\{V_{j_{k}}^{\prime}\right\}_{1 \leq j \leq \ell, 1 \leq k \leq d}$ are algebraically independent over $\widetilde{k}$,

$$
\widetilde{U}=\widetilde{k}\left[\left\{X_{i}^{\prime}\right\}_{1 \leq i \leq m},\left\{Y_{j}^{\prime}\right\}_{1 \leq j \leq \ell},\left\{V_{j_{k}}^{\prime}\right\}_{1 \leq j \leq \ell, 1 \leq k \leq d}\right],
$$

and

$$
\begin{aligned}
\mathfrak{a} \widetilde{U}= & \left(\left\{X_{i}^{\prime}\right\}_{1 \leq i \leq m},\left\{Y_{j}^{\prime}\right\}_{1 \leq j \leq \ell},\left\{V_{j_{k}}^{\prime}\right\}_{1 \leq k \leq d}\right) \cdot\left(\left\{X_{i}^{\prime}\right\}_{1 \leq i \leq m},\left\{Y_{j}^{\prime}\right\}_{1 \leq j \leq \ell}\right) \\
& +\left(V_{j_{k}}^{\prime} V_{j_{k^{\prime}}^{\prime}}^{\prime} \mid 1 \leq j, j^{\prime} \leq \ell, 1 \leq k, k^{\prime} \leq d, j \neq j^{\prime} \text { or } k \neq k^{\prime}\right) \\
& +\left(\frac{Z_{1}}{Z_{k}} V_{j_{k}}^{\prime 2}-Y_{j}^{\prime} \mid 1 \leq j \leq \ell, 1 \leq k \leq d\right) .
\end{aligned}
$$


Let $W=\widetilde{k}\left[\left\{X_{i}^{\prime}\right\}_{1 \leq i \leq m},\left\{V_{j_{k}}^{\prime}\right\}_{1 \leq j \leq \ell, 1 \leq k \leq d}\right]$ in $\widetilde{U}$, and let

$$
\begin{aligned}
\mathfrak{b}= & \left(X_{i}^{\prime}, V_{j_{k}}^{\prime} \mid 1 \leq i \leq m, 1 \leq j \leq \ell, 1 \leq k \leq d\right) \cdot\left(X_{i}^{\prime}, V_{j_{1}}^{\prime 2} \mid 1 \leq i \leq m, 1 \leq j \leq \ell\right) \\
& +\left(V_{j_{k}}^{\prime} V_{j_{k^{\prime}}^{\prime}}^{\prime} \mid 1 \leq j, j^{\prime} \leq \ell, 1 \leq k, k^{\prime} \leq d, j \neq j^{\prime} \text { or } k \neq k^{\prime}\right) \\
& +\left(\frac{Z_{1}}{Z_{k}} V_{j_{k}}^{\prime 2}-V_{j_{1}}^{\prime 2} \mid 1 \leq j \leq \ell, 2 \leq k \leq d\right)
\end{aligned}
$$

in $W$. Then, substituting $Y_{j}^{\prime}$ with $V_{j_{1}}^{\prime 2}$ in $\tilde{U}$ for $1 \leq j \leq \ell$, we get the isomorphism

$$
\widetilde{U} / \mathfrak{a} \widetilde{U} \cong \bar{U}:=W / \mathfrak{b}
$$

of $\tilde{k}$-algebras, under which the prime ideal $\mathfrak{p} \widetilde{U} / \mathfrak{a} \widetilde{U}$ corresponds to the prime ideal $P / \mathfrak{b}$ of $\bar{U}$, where $P=W_{+}:=\left(X_{i}^{\prime}, V_{j_{k}}^{\prime} \mid 1 \leq i \leq m, 1 \leq j \leq \ell, 1 \leq k \leq d\right)$. Then, since $\mathfrak{b}+\left(V_{j_{1}}^{\prime 2} \mid 1 \leq j \leq \ell\right)=P^{2}$ and

$$
\ell_{W_{P}}\left(\left(\mathfrak{b}+\left(V_{j_{1}}^{\prime 2} \mid 1 \leq j \leq \ell\right)\right) W_{P} / \mathfrak{b} W_{P}\right)=\ell
$$

we get

$$
\begin{aligned}
\ell_{\bar{U}_{P}}\left(\bar{U}_{P}\right) & =\ell_{W_{P}}\left(W_{P} / P^{2} W_{P}\right)+\ell_{W_{P}}\left(\left(\mathfrak{b}+\left(V_{j_{1}}^{\prime 2} \mid 1 \leq j \leq \ell\right)\right) W_{P} / \mathfrak{b} W_{P}\right) \\
& =(m+\ell d+1)+\ell
\end{aligned}
$$

Thus, $\ell_{C_{\mathfrak{p}}}\left(C_{\mathfrak{p}}\right)=\ell_{\bar{U}_{P}}\left(\bar{U}_{P}\right)=m+\ell d+\ell+1$.

We have by the associative formula of multiplicity that

$$
\mathrm{e}_{0}(\mathfrak{q})=\ell_{C_{\mathfrak{p}}}\left(C_{\mathfrak{p}}\right) \cdot \mathrm{e}_{0}^{C / \mathfrak{p} C}((\mathfrak{q}+\mathfrak{p} C) / \mathfrak{p} C)=m+\ell d+\ell+1
$$

because $\mathfrak{p}=\sqrt{\mathfrak{a}}$ and $C / \mathfrak{p} C=U / \mathfrak{p}=k\left[Z_{k} \mid 1 \leq k \leq d\right]$. On the other hand, we have

$$
C / \mathfrak{q}=k\left[\left\{X_{k}\right\}_{1 \leq k \leq m},\left\{Y_{j}\right\}_{1 \leq j \leq \ell},\left\{V_{j_{k}}\right\}_{1 \leq j \leq \ell, 1 \leq k \leq d}\right] / \mathfrak{c}^{2}
$$

where

$$
\mathfrak{c}=\left(X_{i}, Y_{j}, V_{j_{k}} \mid 1 \leq i \leq m, 1 \leq j \leq \ell, 1 \leq k \leq d\right) .
$$

Thus, $\mathrm{e}_{0}(\mathfrak{q})=\ell_{C}(C / \mathfrak{q})=m+\ell d+\ell+1$, so that $C$ is a Cohen-Macaulay ring.

Proposition 6.4. We have $\ell_{C}\left(\tilde{J}_{\Lambda} / J_{\Lambda}\right)=\ell, \tilde{J}_{\Lambda}^{2}=\mathfrak{q} \tilde{J}_{\Lambda}$, and $\tilde{J}_{\Lambda}^{n}=J_{\Lambda}^{n}$ for all $n \geq 2$. Here $\tilde{J}_{\Lambda}=\bigcup_{\ell>0}\left[J_{\Lambda}^{\ell+1}: J_{\Lambda}^{\ell}\right]$ denotes the Ratliff-Rush closure of $J_{\Lambda}$ (see [17]). 
Proof. Let $K=J_{\Lambda}+\left(y_{1}, y_{2}, \ldots, y_{\ell}\right)$. Then $\ell_{C}\left(K / J_{\Lambda}\right)=\ell$ and $K^{2}=\mathfrak{q} K=$ $J_{\Lambda}^{2}$. Therefore, $\tilde{K}=K$ because $K^{2}=\mathfrak{q} K$, while we have $\tilde{K}=\tilde{J}_{\Lambda}$ because $K^{2}=J_{\Lambda}^{2}$. We also have $\tilde{J}_{\Lambda}^{n}=\mathfrak{q}^{n-2} \tilde{J}_{\Lambda}^{2}=\mathfrak{q}^{n-2} K^{2}=J_{\Lambda}^{n}$ for all $n \geq 2$. Thus, we get the required assertions.

We are now in a position to finish the proof of Theorem 6.2.

Proof of Theorem 6.2. Because $\widetilde{J_{\Lambda} B}=\tilde{J}_{\Lambda} B$, we get $\ell_{A}\left(\widetilde{J_{\Lambda} B} / J_{\Lambda} B\right)=\ell$ and ${\widetilde{J_{\Lambda} B}}^{2}=\mathfrak{q}\left(\widetilde{J_{\Lambda} B}\right)$ by Proposition 6.4 . Hence, we have

$$
\begin{aligned}
\mathrm{e}_{1}\left(J_{\Lambda} B\right) & =\mathrm{e}_{1}\left(\widetilde{J_{\Lambda} B}\right)=\mathrm{e}_{0}\left(\widetilde{J_{\Lambda} B}\right)-\ell_{B}\left(B / \widetilde{J_{\Lambda} B}\right) \\
& =\mathrm{e}_{0}\left(J_{\Lambda} B\right)-\ell_{B}\left(B / J_{\Lambda} B\right)+\ell_{B}\left(\widetilde{J_{\Lambda} B} / J_{\Lambda} B\right) \\
& =\mathrm{e}_{0}\left(J_{\Lambda} B\right)-\ell_{B}\left(B / J_{\Lambda} B\right)+\ell
\end{aligned}
$$

and $\mathrm{e}_{i}\left(J_{\Lambda} B\right)=\mathrm{e}_{i}\left(\widetilde{J_{\Lambda} B}\right)=0$ for all $2 \leq i \leq d$ by [14, Theorem 2.1]. Since

$$
C / J_{\Lambda} \cong k\left[X_{i}, Y_{j} \mid 1 \leq i \leq m, 1 \leq j \leq \ell\right] / \mathfrak{c}^{\prime},
$$

where $\mathfrak{c}^{\prime}=\left(X_{i}, Y_{j} \mid 1 \leq i \leq m, 1 \leq j \leq \ell\right)^{2}+\left(X_{\alpha} \mid \alpha \in \Lambda\right)$, we have $\ell_{B}(B /$ $\left.J_{\Lambda} B\right)=\ell_{C}\left(C / J_{\Lambda}\right)=m+\ell+1-\sharp \Lambda$. Hence, $\mathrm{e}_{1}\left(J_{\Lambda} B\right)=\sharp \Lambda+\ell d+\ell$ because $\mathrm{e}_{0}\left(J_{\Lambda} B\right)=\mathrm{e}_{0}(\mathfrak{q} B)=m+\ell d+\ell+1$. This gives the proof of assertions (1), $(2),(3)$, and (4).

Assertions (5) and (6). Since $\widetilde{J_{\Lambda} B}{ }^{2}=\mathfrak{q} \widetilde{J_{\Lambda} B}, \mathrm{R}^{\prime}\left(\widetilde{J_{\Lambda} B}\right)$ is a Cohen-Macaulay ring. We put $W^{\prime}=\mathrm{R}^{\prime}\left(\widetilde{J_{\Lambda} B}\right) / \mathrm{R}^{\prime}\left(J_{\Lambda} B\right)$ and look at the exact sequence

$$
0 \rightarrow \mathrm{R}^{\prime}\left(J_{\Lambda} B\right) \rightarrow \mathrm{R}^{\prime}\left(\widetilde{J_{\Lambda} B}\right) \rightarrow W^{\prime} \rightarrow 0\left(*^{\prime}\right)
$$

of graded $\mathrm{R}^{\prime}\left(J_{\Lambda} B\right)$-modules. Observe that $W^{\prime}=\left[W^{\prime}\right]_{1}=\widetilde{J_{\Lambda} B} / J_{\Lambda} B$ because ${\widetilde{J_{\Lambda} B}}^{n}=\left(J_{\Lambda} B\right)^{n}$ for all $n \geq 2$ by Proposition 6.4 , whence we have $\ell_{B}\left(W^{\prime}\right)=\ell$.

Let $N=\left(\mathfrak{n}, \mathrm{R}\left(J_{\Lambda} B\right)_{+}, t^{-1}\right) \mathrm{R}^{\prime}\left(J_{\Lambda} B\right)$ be the unique graded maximal ideal of $\mathrm{R}^{\prime}\left(J_{\Lambda} B\right)$. Then since $\mathrm{R}^{\prime}\left(\widetilde{J_{\Lambda} B}\right)$ is a Cohen-Macaulay ring, applying local cohomology functors $\mathrm{H}_{N}^{i}(*)$ to the exact sequence $\left(*^{\prime}\right)$ yields $\mathrm{H}_{N}^{i}\left(\mathrm{R}^{\prime}\left(J_{\Lambda} B\right)\right)=$ (0) for all $i \neq 1, d+1$, and $\mathrm{H}_{N}^{1}\left(\mathrm{R}^{\prime}\left(J_{\Lambda} B\right)\right)=W^{\prime}$. Then because $\mathfrak{n} W^{\prime}=\mathfrak{n}\left(\widetilde{J_{\Lambda} B} /\right.$ $\left.J_{\Lambda} B\right)=(0)$, we have $\mathfrak{n H}_{N}^{1}\left(\mathrm{R}^{\prime}\left(J_{\Lambda} B\right)\right)=(0)$. Thus, $\mathrm{R}^{\prime}\left(J_{\Lambda} B\right)$ is a Buchsbaum ring with the Buchsbaum invariant

$$
\mathbb{I}\left(\mathrm{R}^{\prime}\left(J_{\Lambda} B\right)\right)=\sum_{i=0}^{d}\left(\begin{array}{l}
d \\
i
\end{array}\right) \ell_{B}\left(\mathrm{H}_{N}^{i}\left(\mathrm{R}^{\prime}\left(J_{\Lambda} B\right)\right)\right)=d \cdot \ell_{B}\left(W^{\prime}\right)=\ell d
$$


whence so is the graded $\operatorname{ring} \mathrm{G}\left(J_{\Lambda} B\right)=\mathrm{R}^{\prime}\left(J_{\Lambda} B\right) / t^{-1} \mathrm{R}^{\prime}\left(J_{\Lambda} B\right)$. We similarly have that $\mathrm{R}\left(J_{\Lambda} B\right)$ is a Buchsbaum ring with $\mathbb{I}\left(\mathrm{R}\left(J_{\Lambda} B\right)\right)=\ell d$, because $\mathrm{R}\left(\widetilde{J_{\Lambda} B}\right)$ is a Cohen-Macaulay ring and $\mathrm{R}\left(\widetilde{J_{\Lambda} B}\right) / \mathrm{R}\left(J_{\Lambda} B\right) \cong \widetilde{J_{\Lambda} B} / J_{\Lambda} B$. This completes the proof of Theorem 6.2 .

Let us now consider the following.

Put $J=J_{\{1,2, \ldots, m\}} B$, and put $A=k+J$. Then $A$ is a local $k$-subalgebra of $B$ with maximal ideal $\mathfrak{m}=J$, and $B$ is a module finite extension of $A$, because $\ell_{A}(B / A)=\ell_{A}(B / J)-1=\ell$. Hence, $A$ is a Noetherian local ring with $\operatorname{dim} A=\operatorname{dim} B=d$ by Eakin-Nagata's theorem (see [15]). We fix a subset $\Lambda$ of $\{1,2, \ldots, m\}$ and put

$$
I=J_{\Lambda} B \quad \text { and } \quad Q=\left(a_{1}, a_{2}, \ldots, a_{d}\right) A .
$$

Then $I$ is an m-primary ideal in $A$ and $Q$ is a parameter ideal in $A$ and a reduction of $I$. We then have the following.

THEOREM 6.5. The following assertions hold true.

(1) Here $A$ is a Buchsbaum local ring with $\mathrm{H}_{\mathfrak{m}}^{i}(A)=(0)$ for all $i \neq 1$, $d$ and $\mathrm{H}_{\mathfrak{m}}^{1}(A) \cong B / A$, whence $\ell_{A}\left(\mathrm{H}_{\mathfrak{m}}^{1}(A)\right)=\ell ;$

(2) $\mathrm{e}_{0}(I)=m+\ell d+\ell+1$;

(3) $\mathrm{e}_{1}(I)=\sharp \Lambda+\ell d+\ell$;

(4) $\mathrm{e}_{i}(I)=0$ for $2 \leq i \leq d-1$ and $\mathrm{e}_{d}(I)=(-1)^{d+1} \ell$;

(5) $2 \mathrm{e}_{0}(I)-\mathrm{e}_{1}(I)+\mathrm{e}_{1}(Q)=2 \ell_{A}(A / I)+\ell_{A}\left(I / I^{2}+Q\right)$;

(6) $\mathrm{G}(I)$ is a Buchsbaum ring with $\mathrm{H}_{M}^{i}(\mathrm{G}(I))=(0)$ for all $i \neq 1, d$, and $\mathrm{H}_{M}^{1}(\mathrm{G}(I))=\left[\mathrm{H}_{M}^{1}(\mathrm{G}(I))\right]_{1} \cong \mathrm{H}_{\mathfrak{m}}^{1}(A) \cong B / A$.

Before giving the proof of Theorem 6.5, let us note the following.

Lemma 6.6. We have $\left[\left(a_{1}, a_{2}, \ldots, \check{a_{i}}, \ldots, a_{d}\right) A:_{A} a_{i}\right] \subseteq I$ for all $1 \leq i \leq d$, whence condition $\left(\mathrm{C}_{2}\right)$ is satisfied for the ring $A$ and the ideals $I$ and $Q$.

Proof. Take $x \in\left[\left(a_{1}, a_{2}, \ldots, \check{a}_{i}, \ldots, a_{d}\right) A:_{A} a_{i}\right]$, then $a_{i} x \in\left(a_{1}, a_{2}, \ldots, \check{a}_{i}\right.$, $\left.\ldots, a_{d}\right) A \subseteq\left(a_{1}, a_{2}, \ldots, \check{a}_{i}, \ldots, a_{d}\right) B$. Then since $B$ is a Cohen-Macaulay ring and $a_{1}, a_{2}, \ldots, a_{d}$ forms a system of parameters for $B$, we have $x \in\left(a_{1}, a_{2}\right.$, $\left.\ldots, \check{a}_{i}, \ldots, a_{d}\right) B \subseteq J_{\Lambda} B=I$, as required.

Proof of Theorem 6.5. Assertion (1). Look at the canonical exact sequence

$$
0 \rightarrow A \rightarrow B \rightarrow B / A \rightarrow 0
$$


of $A$-modules. Then since $B$ is a Cohen-Macaulay ring and $\ell_{A}(B / A)=$ $\ell_{A}(B / J)-1=\ell$, we get $\mathrm{H}_{\mathfrak{m}}^{i}(A)=(0)$ for all $i \neq 1, d$ and $\mathrm{H}_{\mathfrak{m}}^{1}(A) \cong B / A$. Because $\mathfrak{m} B=J \subseteq A$, we have $\mathfrak{m H}_{\mathfrak{m}}^{1}(A)=(0)$, whence $A$ is a Buchsbaum local ring (see [20, I. Proposition 2.12]).

Assertions (2), (3), and (4). Since $\ell_{A}(B / A)=\ell_{A}(B / J)-1=\ell, \operatorname{dim} A=$ $\operatorname{dim} B=d \geq 2$, and

$$
\ell_{A}\left(A / I^{n+1}\right)=\ell_{A}\left(B / J_{\Lambda}^{n+1} B\right)-\ell_{A}(B / A)
$$

for all $n \geq 0$, we have $\mathrm{e}_{0}(I)=\mathrm{e}_{0}\left(J_{\Lambda} B\right)=m+\ell d+\ell+1, \mathrm{e}_{1}(I)=\mathrm{e}_{1}\left(J_{\Lambda} B\right)=$ $\sharp \Lambda+\ell d+\ell, \mathrm{e}_{i}(I)=\mathrm{e}_{i}\left(J_{\Lambda} B\right)=0$ for $2 \leq i \leq d-1$, and $\mathrm{e}_{d}(I)=\mathrm{e}_{d}\left(J_{\Lambda} B\right)+$ $(-1)^{d+1} \ell_{A}(B / A)=(-1)^{d+1} \ell$ by Theorem 6.2 .

Assertion (5). Because $I^{2}+Q=\mathfrak{q} B$, we have

$$
\begin{aligned}
\ell_{A}\left(A / I^{2}+Q\right) & =\ell_{A}\left(B / I^{2}+Q\right)-\ell_{A}(B / A) \\
& =\ell_{A}(B / \mathfrak{q} B)-\ell_{A}(B / A) \\
& =\{m+\ell+\ell d+1\}-\ell \\
& =m+\ell d+1 .
\end{aligned}
$$

Then because $\ell_{A}(A / I)=\ell_{A}\left(B / J_{\Lambda} B\right)-\ell_{A}(B / A)=m+1-\sharp \Lambda$ and $\mathrm{e}_{1}(Q)=$ $-h^{1}(A)=-\ell_{A}(B / A)=-\ell$ by $\left[19\right.$, Korollar 3.2], we get $2 \mathrm{e}_{0}(I)-\mathrm{e}_{1}(I)+$ $\mathrm{e}_{1}(Q)=2 \ell_{A}(A / I)+\ell_{A}\left(I / I^{2}+Q\right)$, as required.

Assertion (6). The ring $\mathrm{G}(I)$ is a Buchsbaum ring with $\mathrm{H}_{M}^{i}(\mathrm{G}(I))=(0)$ for all $i \neq 1, d$ and $\mathrm{H}_{M}^{1}(\mathrm{G}(I))=\left[\mathrm{H}_{M}^{1}(\mathrm{G}(I))\right]_{1} \cong B / A$ by assertions $(1)$ and (5), Lemma 6.6, and Theorem 1.1. This completes the proof of Theorem 6.5.

\section{REFERENCES}

[1] A. Corso, Sally modules of $\mathfrak{m}$-primary ideals in local rings, Comm. Algebra 37 (2009), 4503-4515. MR 2588863. DOI 10.1080/00927870802266490.

[2] A. Corso, C. Polini, and W. V. Vasconcelos, Multiplicity of the special fiber of blowups, Math. Proc. Cambridge Philos. Soc. 140 (2006), 207-219. MR 2212275. DOI 10.1017/ S0305004105009023.

[3] J. Elias and G. Valla, Rigid Hilbert functions, J. Pure Appl. Algebra 71 (1991), 19-41. MR 1107650. DOI 10.1016/0022-4049(91)90038-4.

[4] L. Ghezzi, S. Goto, J. Hong, K. Ozeki, T. T. Phuong, and W. V. Vasconcelos, Variation of the first Hilbert coefficients of parameters with a common integral closure, J. Pure Appl. Algebra 216 (2012), 216-232. MR 2826435. DOI 10.1016/j.jpaa.2011. 06.008 .

[5] S. Goto, Noetherian local rings with Buchsbaum associated graded rings, J. Algebra 86 (1984), 336-384. MR 0732255. DOI 10.1016/0021-8693(84)90037-1. 
[6] S. Goto and K. Nishida, Hilbert coefficients and Buchsbaumness of associated graded rings, J. Pure Appl. Algebra 181 (2003), 61-74. MR 1971805. DOI 10.1016/ S0022-4049(02)00325-0.

[7] S. Goto, K. Nishida, and K. Ozeki, Sally modules of rank one, Michigan Math. J. 57 (2008), 359-381. MR 2492458. DOI $10.1307 / \mathrm{mmj} / 1220879414$.

[8] S. Goto and K. Ozeki, The structure of Sally modules-towards a theory of nonCohen-Macaulay cases, J. Algebra 324 (2010), 2129-2165. MR 2684135. DOI 10. 1016/j.jalgebra.2010.07.017.

[9] - "Uniform bounds for Hilbert coefficients of parameters" in Commutative Algebra and Its Connections to Geometry (Olinda, 2009), Contemp. Math. 555, Amer. Math. Soc., Providence, 2011, 97-118. MR 2882677. DOI 10.1090/conm/555/ 10992.

[10] S. Goto and Y. Shimoda, On Rees algebras over Buchsbaum rings, J. Math. Kyoto Univ. 20 (1980), 691-708. MR 0592354.

[11] A. Guerrieri and M. E. Rossi, Hilbert coefficients of Hilbert filtrations, J. Algebra 199 (1998), 40-61. MR 1489353. DOI 10.1006/jabr.1997.7194.

[12] S. Huckaba and T. Marley, Depth formulas for certain graded rings associated to an ideal, Nagoya Math. J. 133 (1994), 57-69. MR 1266362.

[13] C. Huneke, On the symmetric and Rees algebra of an ideal generated by a d-sequence, J. Algebra 62 (1980), 268-275. MR 0563225. DOI 10.1016/0021-8693(80)90179-9.

[14] - Hilbert functions and symbolic powers, Michigan Math. J. 34 (1987), 293318. MR 0894879. DOI 10.1307/mmj/1029003560.

[15] M. Nagata, A proof of the theorem of Eakin-Nagata, Proc. Japan Acad. Ser. A Math. Sci. 67 (1991), 238-239. MR 1137916.

[16] K. Ozeki, The equality of Elias-Valla and the associated graded ring of maximal ideals, J. Pure Appl. Algebra 216 (2012), 1306-1317. MR 2890504. DOI 10.1016/j. jpaa.2012.01.004.

[17] L. J. Ratliff and D. E. Rush, Two notes on reductions of ideals, Indiana Univ. Math. J. 27 (1978), 929-934. MR 0506202.

[18] M. E. Rossi and G. Valla, Hilbert Functions of Filtered Modules, Lect. Notes Unione Mat. Ital. 9, Springer, Berlin, 2010. MR 2723038. DOI 10.1007/978-3-642-14240-6.

[19] P. Schenzel, Multiplizitäten in verallgemeinerten Cohen-Macaulay-Moduln, Math. Nachr. 88 (1979), 295-306. MR 0543409. DOI 10.1002/mana.19790880122.

[20] J. Stückrad and W. Vogel, Buchsbaum Rings and Applications: An Interaction between Algebra, Geometry and Topology, Springer, Berlin, 1986. MR 0881220.

[21] N. V. Trung, Toward a theory of generalized Cohen-Macaulay modules, Nagoya Math. J. 102 (1986), 1-49. MR 0846128.

[22] - Reduction exponent and degree bound for the defining equations of graded rings, Proc. Amer. Math. Soc. 101 (1987), 229-236. MR 0902533. DOI 10.2307/ 2045987.

[23] W. V. Vasconcelos, "Hilbert functions, analytic spread, and Koszul homology" in Commutative Algebra: Syzygies, Multiplicities, and Birational Algebra (South Hadley, Mass., 1992), Contemp. Math. 159, Amer. Math. Soc., Providence, 1994, 401-422. MR 1266195. DOI 10.1090/conm/159/01520. 
Department of Mathematical Science Faculty of Science

Yamaguchi University

1677-1 Yoshida

Yamaguchi 753-8512

Japan

ozeki@yamaguchi-u.ac.jp 